Aus der chirurg. Universitätsklinik Basel (Direktor: Professor F. de Quervain).

\title{
Der Kompressionsbruch und die traumatische Erweichung des Mondbeines.
}

Von Dr. Georges Guye, Assistenzarzt von Dr. Rollier in Leysin.

(Mit I 7 Abbildungen).

Unter den verschiedenen Verletzungen des Handgelenks, welche seit der Röntgenuntersuchung aus dem Gebiete der un. bestimmten Handgelenksdistorsion in dasjenige der scharf umschriebenen Skelettverletzungen übergegangen sind, zeichnet sich eine Form zwar nicht durch ihre große Häufigkeit, aber durch ihren trotz des Röntgenbildes noch einigermaßen rätselhaften Charakter aus: Es ist dies die Kompressionsfraktur bzw. die traumatische Erweichung des Mondbeines. Diese beiden Bezeichnungen weisen uns ohne weiteres darauf hin, wie verschieden diese Skelettveränderung von den verschiedenen Autoren noch aufgefaßt wird. Waren auch vor I 904 schon einige hierher gehörige Röntgenbilder beschrieben worden, so dürfte doch der erste klinisch, bakteriologisch und histologisch nach allen Richtungen hin untersuchte Fall derjenige scin, den Prof. de Quervain in seiner damaligen chirurgischen Abteilung des Spitals in La Chaux-de-Fonds 1904 beobachtete, und mir mit drei weiteren, nichtoperierten Fällen aus seiner dortigen Tätigkeit zur Bearbeitung übergab. Äußere Umstände haben die Fertigstellung dieser Arbeit in unerwünschter Weise verzögert. Unterdessen hat Prof. de Quervain noch einen weiteren Fall beobachtet und operiert, den er mir ebenfalls überwies. Dazu kommt eine ihm von Herrn Dr. Ruppanner, Spitalarzt in Samaden, zur Verfügung gestellte Beobachtung (ebenfalls operiert), und ein in der chirurgischen Poliklinik in Basel zum Zwecke der Begutachtung vom leitenden Arzte derselben, Herrn Dr. Is e- 
lin, untersuchter Fall, so daß wir im ganzen über 7 neue Fälle verfügen. Mittlerweile haben sich nun auch von anderen Seiten her die Mitteilungen über derartige Fälle vermehrt, und es ist in letzten Jahr auch ein erster histologischer Befund in der Literatur veröffentlicht worden (Ba um). In den Lehrbüchern der Chirurgie - mit Ausnahme der chirurgischen Diagnostik von de Quervain -- wird dicse Verletzung bis jetzt nicht erwähnt. Zur sicheren Deutung des klinischen und Röntgenbefundes fehlten bis jetzt unter anderem zwei sehr wesentliche Momente: die histologische Untersuchung von Schnitten durch den ganzen exstirpierten Knochen und ferner eine sich über Jahre crstreckende Beobachtung operierter und nichtoperierter Fälle. Nach diesen beiden Seiten hin verfügen wir über Beobachtungen, welche zur Klärung noch offener Fragen beitragen werden.

\section{Ligene Fülle.}

Wir beginnen mit der Beschreibung unserer eigenen Fälle und geben die Krankengeschichten derselben in chronologischer Reihenfolge wieder.

Fall x. Frau M. D., 24 Jahre alt (Privatbeobachtung von Prof. de Quervain). Dic sonst gesunde Patientin, deren Familienanamnese keine sichere, und deren persönliche Anamnese gar kcine Andeutungen von tuberkulösen Erkrankungen aufweist, begann im Anschluß an eine heftige Bewegrung, wahrscheinlich mit Dorsalflexion im rechten Handgelenk, in demsclben Schmerzen bei Bewegungen zu verspüren, die sich angeblich mehr auf die Radialseite desselben lokalisierten. Objcktiv fand sich 3 Monate nach der erwähnten Beweg'ung (4. VII. I902) leichte Schwelinng (Umfangsvermehrung $3 / 4 \mathrm{~cm}$ ), Druckempfindlichkeit sowohl volar wie dorsal vor dem Radiuscnde, leichte Kapselverdickung auf der Dorsalseite des Handgelenks, mit Andeutung von Flussigkcitserguß. Die Volarflexion fand sich leicht, dic Dorsalflexion stark gehemmt.

Das Röntgenbild (Fig. I) ergab allgemcine leichte Osteoporose des Handgelenks und eine leichte Verkürzung des Lunatum in proximo-distaler Richtung, bei abnormer, etwas höckeriger Begrenzung, Verdichtung und abnormer Strukturzeichnung des proximalen Anteils. Die übrigen Knochen zeigten sich normal.

Da damals die uns hier beschäftigende Verletzung des Lunatum noch nicht bekannt war, so wurde mit Wahrscheinlichkeit eine tuberkulöse Erkrankung angenommen, und es wurden von Juli bis Oktober 1902 einige Karbol-Jodoforminjektionen vorgenommen. Eine im 
Dezember ausgeführte Nachuntersuchung zeigte erhebliche Besserung der I unktion, so daß dic Paticntin sich nicht mehr zur Untersuchung stellte. Eine 5 Jahre später vorgenommene zweite Nachuntersuchung crgab für Palpation und Funlitionsprüfung normale Verhältnisse. Allerdings wurde damals kein Röntgenbild aufgenommen.

Eine weitere Nachuntersuchung im Januar I9I4, also beinahe 12 Jahre nach Beginn des Leidens, ergab folgenden Befund:

Dic rechte Hand erscheint etwas kürzer als die linke und das Handgelenk etwas verdickt (I cm Umfangsvermchrung), bei der

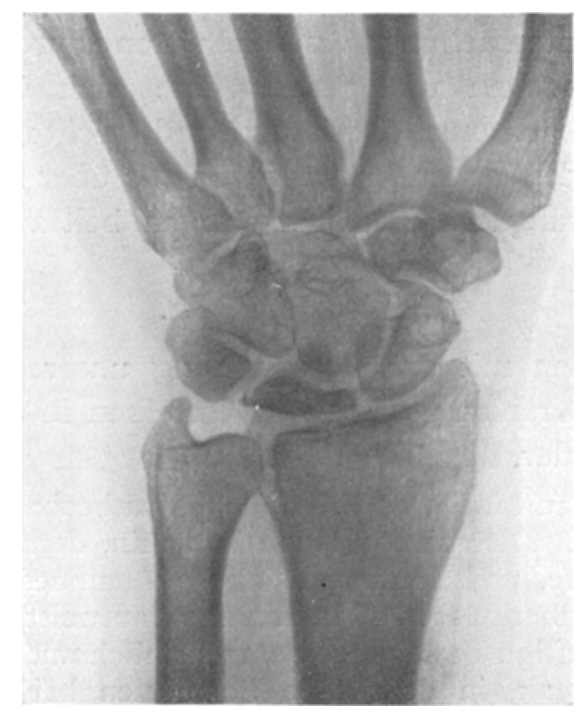

Fig. I. Fall r. I. Aufnahme Juli rgoz. Veränderungen auf das Lunatum beschränkt.

Palpation zcigt sich die normal vor der dorsalen Radiuslippe vorhandene Einsenkung beinahe verstrichen. Die Palpation ist dabei auf der Dorsalseite etwas schmerzhaft. Die Dorsalflexion geht bis zu I 35 Grad, die Volarflexion bis zu 550 Grad. Ad- und Abduktion sind weniger eingeschränkt. Dic Fingerbewegungen sind frei, abcr etwas schwächer als links. Dic Patientin verspürt Schmerzen im Ifandgelenk bei starken Muskelanstrengungen, besonders im Sinne der Pro- und Supination, so z. B. beim Auswinden der Wäsche. Sie besorgt aber immerhin alle ihre häuslichen Arbeiten.

Die beinahe 12 Jahre nach Bcginn der Erkrankung aufgenommenen Röntgenbilder (Fig. 2--4) zeigen, daß die Veränderung des Lunatum deutlich zugenommen hat. Dasselbe ist in zwei Teile ge- 


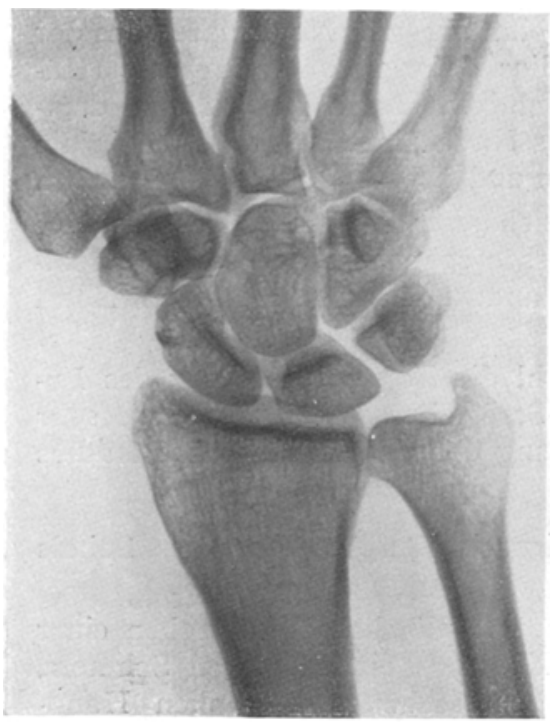

Irig. 2.

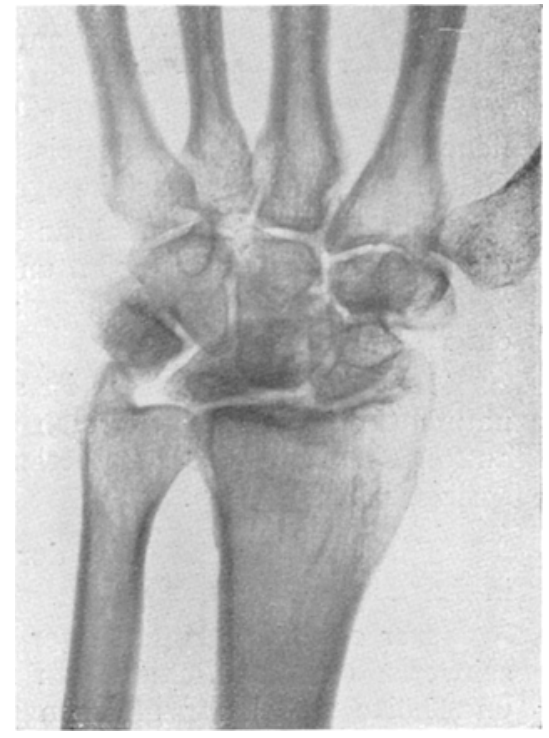

Iig. 3

Fig. 2. Fall r. Gesunde Seite. Keine Spur von Arthritis defomans.

Fir. 3. Fall I. Aufnahme vom Januar I9I4 (also II $1 / 2$ Jahr nach der Aufnahme von Firr. I.) Deutliche Arthritis deformans in der ganzen Umgebung des Lunatum. Das letztere aus dem Radiocarpalgelenk herausgedrän!, nach Fior. 4 in ein dorsales und ein volares Sitick getrennt.

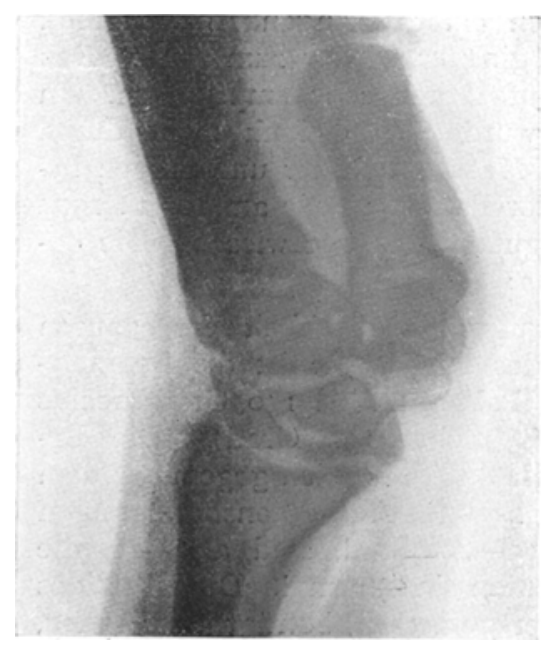

Fig. 4. Fall 1. Aufnahme rom 4. Jan. IgI4 im Profil.

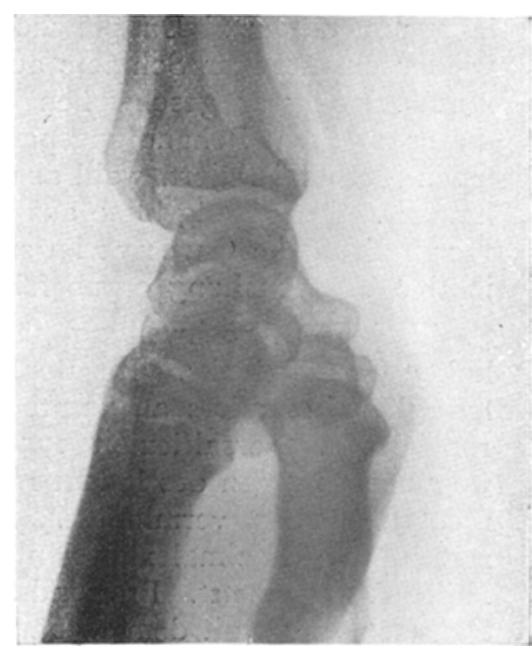

ligs. 5. Gesunde Seite desselben Falles. 
trennt, einem volaren und einem dorsalen. Uberdies sind an den benachbarten Skelettcilen, so am Capitatum, am Naviculare, an der Ulna, ganz besonders aber am Radius deformierende Prozesse (Unregelmäßigkeit der Gelenkfläche) sichtbar. Auch besteht eine deutliche Verdünnung des Knorpels. Das Naviculare hat sich etwas in die Gelenkfläche des Radius cingegraben und überdies völligr steil gestellt.

Auch jetzt noch beschränken sich aber die Veränderungen auf die Umgebung des Lunatum und lassen schon dic Carpo-Metacarpalgrelenke völlig frei. Ebenso ist das Handwurzelskelett der linken Hand cin völlig normales.

In der Profilaufnahme ist sowohl dic Formveränderung des Lunatum (Zweiteilung!) wic auch die Stcilstellung des Naviculare sehr auffallend. Auch die Formveränderung der Gelenkfläche des Radius springt in die Augen.

Ii all 2. A. E. (Aus der chirurgischen Abteilung des Spitals in La Chaux-de-Fonds.)

Der 26jährige Handlanger verspürte plötzlich beim Heben einer schweren eisernen Schiene am I7. XI. 1903 einen starken Schmerz, und hörte dabei zur glcichen Zcit ein Knacken im linken Hand. gelenk. Er arbeitete noch während drei Tagen weiter, trotz der Anschwellung und der Schmerzen im verletzten Gelenk, und beriet dann einen Arzt. Die eingeleitete Behandlung brachte jedoch keine wesentliche Besserung. Die Bewegungen im Handgelenk blieben beschränkt und schmerzhaft. Auf ärztlichen Rat hin fing er nach Io Tagen zu arbeiten an. Die abendliche Schwellung verschwand in der Regel während der Nacht. Der Zustand blicb aber drei Monate lang unverändert. Da plötzlich, nach einer geringen Anstrengung, wicder cin heftiger Schmerz auftrat, ging er wieder zum Arzte. Das Handgelenk war geschwollen und auf Druck sehr empfindlich. In dieser zweiten Behandlungsperiode wurde das Gelenk nicht durch einen Verband fixiert, wohl aber mit Massage, Bäder und Salben behandelt. Der Erfolg blicb aus und der Patient wurde am ro. V. 1904 Herrn Prof. de Quervain zur Begutachtung überwiesen. Die objektive Untersuchung crgab folgendes:

Leichte Anschwcllung und Druckempfindlichkeit des ganzen linken Handgelenks. Im Bereiche des Naviculare ist die Schmerzhaftigkeit besonders ausgesprochen. Palmarflexion 140 Grad (rechts I20 Grad), Dorsalflexion 175 Grad (rechts I30 Grad). Die Bewegungsexkursion des linken Ilandgelenks ist folglich gegenüber dem rechten um 65 vermindert. Dic Ab- und Adduktionsbewegungen zeigen cine Minimalexkursion von 15-20 Grad, sind also um die Hälfte herabgesctzt. Pro- und Supination sind normal. Dic Kraft des Faustschlusses, mit dem Dynamometer gemessen, beträgt links $15 \mathrm{~kg}$, rechts $50 \mathrm{~kg}$. 
Die Röntgcnuntersuchung (Fig. 6) ergibt:

I. Allgemeine Osteoporose leichten Grades.

2. Verkürzung der Distanz zwischen der Basis der rechten Metacarpalknochen und des Processus styloidcus radii um $\mathrm{I} \mathrm{cm}$.

3. SteilsteIlung des an sich unverletzten Naviculare.

4. Das Os lunatum ist hochgradig verändert. Ein dunkler Kern ohne scharfe Strukturzcichnung liegt in einer radius- und triquetrumwärts deutlich erkennbaren, zum Teil abgelösten Knochenschale. Der Durchmesser des Knochens in der Längsrichtung des Gliedes ist verkürzt.

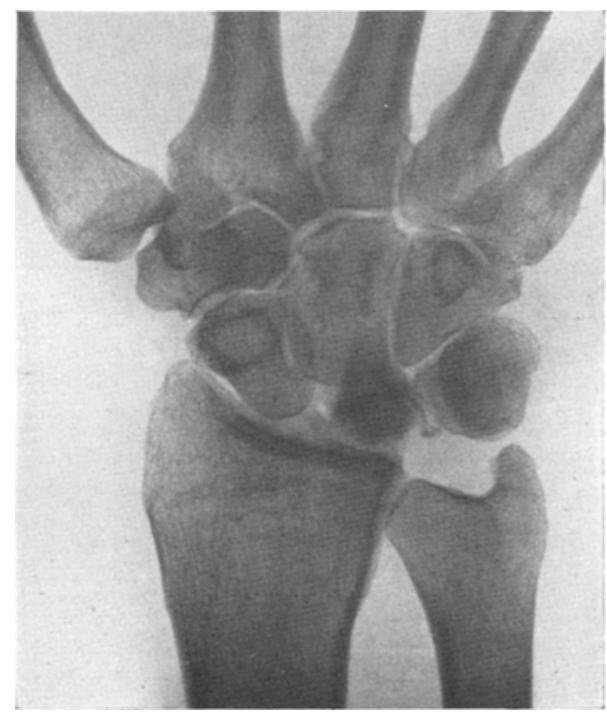

Fig. 6. Fall 2. D)as Lunatum zeigt einen dunkeln (nekrotischen) Kern und eine dünne Knochenschale.

Diagnose: Kompressionsfraktur des Os lunatum mit deformierender Ostitis.

Da man nicht sofort zur opcrativen Entfernung des verletzten Knochens schreiten wolle, wurde versucht, mittels Mechanotherapie die Bewegungsexkursion des IIandgelenks zu verbessern. Diese Behandlung wurde regselmäßig während eines Monats durchgeführt. Der Ërfolg erwies sich aber als ungenügend; die Beweglichkeit des Gelenks besserte sich kaum und die Schmerzen hielten an. Es wurde deshalb am 6. VII. I904 die Exstirpation des Mondbeins vorgenommen.

Operation: Bromäthyläthernarkose. - Esmarchsche Blutleere. Dorsoradialer Längsschnitt. Eröffnung des Gelenks im Be- 
reiche des Lunatum. Die Kapsel ist nicht merklich veründert, die Synovialis ist etwas injiziert. Makroskopisch kcinc Anhaltspunkte für Tuberkulose. Synovia kaum vermehrt, normal ausschend. Der dorsale Teil des Lunatum zcigt Veränderungen im Sinne beginnender deformicrender Ostitis, ebenso die bcnachbarten Teile des Naviculare und des Capitatum (Atrophie des Knorpels). Das Lunatum wird umschnitten. Beim Versuch, dasselbe herauszuheben, kommen nur die dorsalen zwei Drittel mit, ohne daß irgendein gewaltsamer Akt stattgefunden hätte. Der volare Teil wird nachträglich bei starker Volarflexion entfernt. Dic Spongiosa erscheint zwischen den beiden Teilen des Knochens zerstört. Diejenige des dorsalen größeren Stückes sicht größtenteils sklerotisch aus. Diese sklerotische Partic ist etwas gelblich verfärbt. Sic hängt mit der übrigen Spongiosa fest zusammen. Verkäsung oder Granulationsbildung findet sich nirgends. Das Naviculare zeigt keine Andeutung von Fraktur. Dagegen erscheint es infolge der Volumveränderung des Lunatum etwas steil gestellt. Seine distale Knorpelfläche ist normal. Am Triquetrum nichts besonderes. Glasdrain, Naht, Verband in Dorsalflexion.

Am I3. VII. Entfernung der Wundklammern. Verband in starker Dorsalflexion.

Vom I6. VII. an wird der Arm täglich clektrisiert. Es lassen sich durch Elektrisieren alle Bewegungen im linken Handgelenk in normaler Exkursion erziclen.

Am I7. VII. Entlassung. Von da an ambulante Behandlung. Ubungen am Apparat, Faradisation.

Bakteriologischer Befund (Mcerschweinchenimpfung von Synovialis und Knochen. Bakteriologisches Institut Bern, Prof. Tave1): Keine Tuberkulose. Der für 'Tuberkulose ebenfalls negative histologische Befund wird spätcr mitgeteilt werden.

$\mathrm{Nach}$ antersuch ung vom 5. X. : Handgelenk ohne entzündliche Reaktion. Hauttemperatur normal. Keine Schmerzhaftigkeit auf Iruck. Zug in der Achse ist schmerzlos, StoB hingegen leicht schmerzhaft. Funktionspriifung: Palmarflexion I60 Grad, Dorsalflexion 150 Grad. Ab- und Adduktion beschränkt. Pro- und Supination ganz frei. Kraft der Flexion I $5 \mathrm{~kg}$ (50 rechts), Verminderung der Arbeitsfähigkeit mit Rücksicht auf den Beruf des E. auf 20 Proz. geschätzt.

$\mathrm{Nachuntersuchung}$ vom I 5. III. I9I : A. E. gibt an, daB er seit der Operation keine Beschwerden mehr im Handgelenk verspürt hätte. Seit Oktober 1904 hatte er als Taglöhner gearbeitet; allerdings wurde er nie mehr mit schweren Arbeiten betraut. Es ist ihm jedoch möglich, Holz zu sägen und zu spalten; er kann stundenlang mähen; cr hat auch hic und da schwere Lasten getragen, ohne je eine Störung von seiten des Gelenks zu bemerken.

Die objektive Untersuchung ergab folgendes Resultat: Die Ge- 
lung desselben. Er versuchte weiter zu arbeiten. Da aber die Schmerzen und die Schwcllung immer mehr zunahmen, berict er nach 2 Tagen cinen Arzt, welcher cine Verstauchung des linken Handgelenks und eine Tendenosynovitis diagnostizierte. Die Behandlung bestand in Jodtinktureinpinselungen und Massage und dauerte it Tage. Kurze Zeit schon nach der Arbeitsaufnalume traten die gleichen Beschwerden wieder auf, so daß nach cinem Monat die Fand noch gebrauchsunfähig war. Der Patient wurde am 20. V. Iferrn Prof. de Q uervain zur Begutachtung überwiesen. l)ie Untersuchung ergab folgendes Resultat:

Das linke Handgelenk ist diffus leicht verdickt. Man sicht aber keinen abnormen Knochenvorsprung und keine Verschiebung der Carpalknochen. Die auf Druck vorhandene Schmerzhaftigkeit ist auf die Gegend des Processus styloideus ulnae lokalisiert. Zug in der Richtung der Tängsarhse ist schmerzlos, hingegen ist der StoB selur schnerzhaft.

Funktionsprüfung: 1) ie Dorsalflexion beträgt links wie rechts I too Grat. Die Volarflexion ist links glcich 140 Grad, recluts I50 (irad. Die Ab- und Adduktionsbewegungen sind beiderseits etwas beschränkt. Die Fingerbewegungen sind ungestört. Dic Kraft des Faustschlusses am Dynamometer gemessen beträgt rechts $70 \mathrm{~kg}$, links $35 \mathrm{~kg}$.

Röntgenuntersuchung (Fig. 8): Das Lunatum ist auffallend abgeplattet und deformicrt. Das stereoskopische Bild läßt cine deutliche Trennung zwischen dem dorsalen IIorn und dem volaren Teile des Knochens erkennen. Es besteht ein leichter Cirad von Atrophic. Andere Knochenveränderungen sind nicht zu erkennen.

I) iagnose: Kompressionsfraktur des Os lunatum.

Es wurde Wiederaufnahme der Arbeit und neue Begutachtung nach 2-3 Monaten vorgeschlagen. Für die Zwischenzeit wird eime Verminderung der Arbeitsfähigkeit von 25 Proz. angenommen. Eine Nachuntersuchung fand am 6. IX. statt: Lecichtes Öden des Handrückens und ziemlich starke Druckempfindlichkeit in der Gegend des Os lunatum. Die Beweglichkeitseinschränkung ist die glciche wie bei der vorigen Untersuchung.

Auf Grund dieses Ergcbnisses und des Verlaufes der Unfallfolgen lautete die Begutachtung folgendermaBen: Absolute Arbcitsunfähigkeit vom Tage des [ nfalls (8. IV. 1907) bis zum 3r. V. Verminderung der Arbeitsfähiglseit um 25 Proz. von da an und während 8 Monaten. Endlich Verminderung der Arbeitsfähigkeit von I 5 Proz. während vier weiterer Monate, ohne Annahme cines bleibenden Nachteils. Dieser Vorsehlag wurde von dem Verunfallen sowohl als von der Versicherungsgesellschaft angenommen.

Seither konnte der Verunfallte nicht mehr ausfindig gemacht werden. 
gend der Carpalknochen von der Mitte aus nach radial scheint etwas abgeflacht zu sein, d. h. der Vorsprung des Naviculare ist nicht zu fühlen und ist durch eine leichte Depression ersetzt. Die Schne des Extensor indicis proprius ist stark vorspringend. Die $8 \mathrm{~cm}$ lange Narbe ist reaktionslos und auf der Unterlage leicht verschieblich. Der Umfang des Handgelenks unterhalb der Griffelfortsätze beträgt links 18,5 , rechts $18 \mathrm{~cm}$. Die Distanz vom Kopf des Metacarpus III bis Processus styloideus radii ist links 9,5 , rechts $10 \mathrm{~cm}$. Der Umfang des Vorderarms $3 \mathrm{~cm}$ oberhalb des Gelenks ist links 17, rechts $18 \mathrm{~cm}$. Der Zug in der Richtung der Achse sowie der Stoß sind absolut schmerzlos; ebenso starker Druck auf das Gelenk. Funktionsprüfung: Dic Kraft des Faustschlusses ist links glcich wie rechts. Die Fingerbeweglichkeit ist beidcrseits gleich. Pro- und Supination normal. Die Radialadduktion ist rechts ebenso ausgiebig wie links.

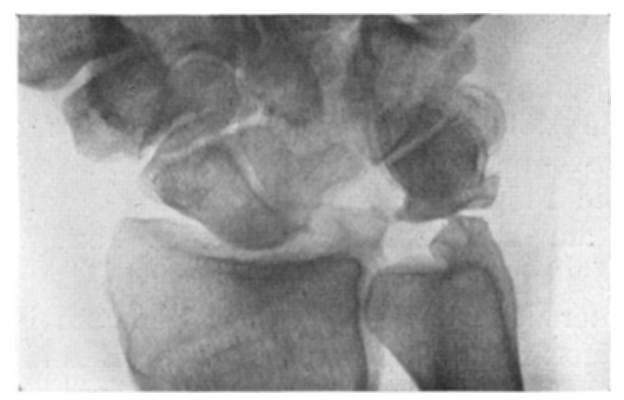

Fig. 7. Fall 2. Io Jahre nach der Operation (März 19I4). Deformierende Veränderungen in der Umgebung der Mondbeinlücke. Die übrigen Gelenke normal (ebenso das rechte Handgelenk).

Die Ulnaradduktion hingregen ist links um 5 Grad geringer als rechts. Dic Dorsalflexion ist links und rechts gleich 150 Grad, die Palmarflexion links I40 Grad, rechts 115 Grad.

Bci einer Nachuntersuchung am 4. III. I9I4, die für die Flexion und die Extension cin etwas weniger günstiges Resultat ergibt, sagt E., er verspüre bei gewissen drehenden Bewegungen bisweilen einen Schmerz im Handgclenk. Er hätte durch den Unfall cine Lohnverminderung von ca. 16 Proz. erlitten.

Die Röntgenuntersuchung (Fig. 7) ergibt die deutlichen Zeichen deformierender Arthritis in der Umgcbung der durch das Lunatum gelassenen Lücke.

Fall 3 (aus der Privatpraxis von Herm Prof. de Quervain). Am 8. IV. 1907 fiel der 25 jährige Küfer A. B. aus einer Höhe von $1,5 \mathrm{~m}$ auf die dorsalflektierte linke Hand. Dabei verspürte er heftige Schmerzen im Handgclenk und bemerkte eine sofortige Schwel- 
Fall 4. E. N., 30 Jahre alt. Schmerzen im linken Ilandgelenk im Anschluß an cinc bei der Arbeit zustande gekommene Distorsion. Lunatum etwas verkürzt in proximodistaler Richtung, kalkhaltiger als die Nachbarknochen, mit eingen kleinen helker sezeichneten Lücken. Begrenzung nach dem Radius hin etwas unregelmäßigr. Der Palient tritt nach mehrwöhiger l3cobachtungsacit atus tem Spitale aus und stellt sich nicht mehr zur Nachunersuchung. Es kann aber in Erfahrung gebracht werden, dab er seiner nicht leichten Arbeit als Heizer seither (bis März 1014) ohne Linterbrechung vorgcstanden hat. Der damals geaußerte Verdacht auf Tuberkulose ist jedenfalls damit olne wciteres hinfiillig gewor-

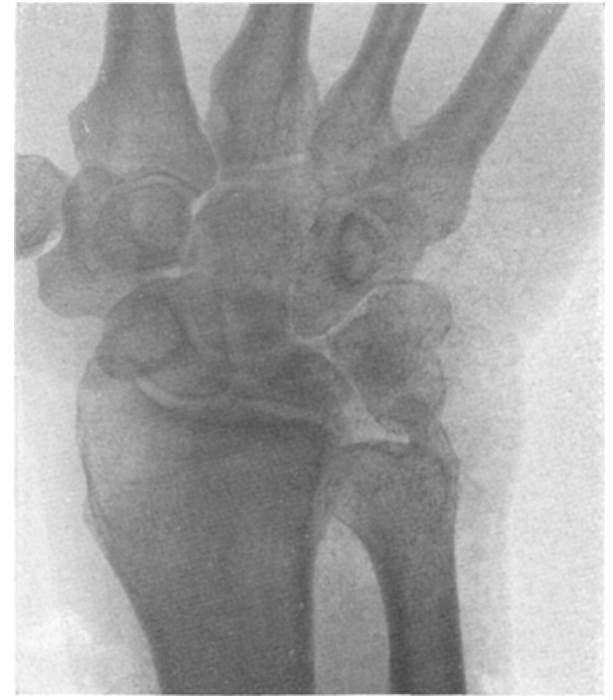

Iig. 8 . den und der fiall roiht sich in typischer Weise in die analogen Verletzungen des Lunatums cin (lig. y).

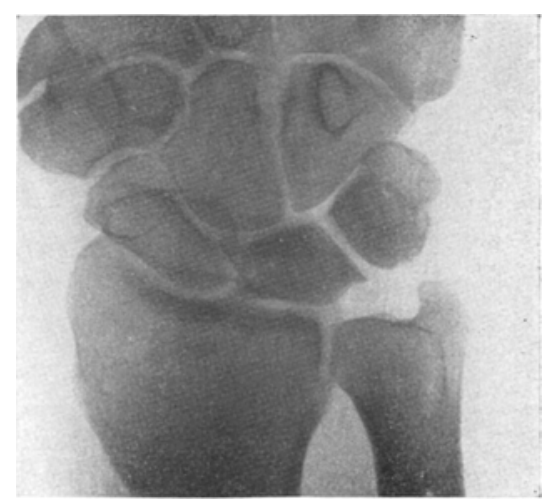

Iig. 9.

Fig. 8. Fall 3. Mässig starke Kompression des Iunatums.

Fig. 9. Fall 4. Mäßige Veränderungen des I.unatum. (Verdichtungr mit kleinen lïcken. liorm unregelmäßig.)

Fall 5 (aus der chirurgischen Klinik und Poliklinik zu Basel). Frau M. aus S., 33 Jahre alt. Im August 1 go 8 beim Öffnen einer mit cinem Schraubendeckel versehenen Fruchtzonservenflasche, wobei die fassende: Hand stark radialwärts gedreht und leicht dorsalflektiert wurde, lïste sich plötzlich der I)eckel. Im gleichen Moment verspürte die Patientin einen mäßigen, aber doch deutlichen Schmerz in der Handgelenkgegend. Dieser verschwand nach cinigen Stunden, so daß die Patientin ihre Hausarbeit wieder weiter verrichten konnte. Einige Tage später aber traten sehr heftige Schmerzen auf und war eine Schwollung auf dem IIandrüicken zu schen. Wïhrend 
zwei Tagen war es der Patientin unmöglich die geringste Bewegung im Handgelenk zu machen. Nach und nach aber verschwand der Schmerz und, obschon die Schwellung immer noch bestand, fing sie an ihre Hand wieder zu gebrauchen. So verflossen 6 Monate, ohne daß sich der Zustand gebessert hätte. Dann konsultierte sie einen Arzt nach dom andern. Die Verletzung wurde zuerst als Verstauchung aufgefaßt und dementsprechend mit Salben und Massage und Jodtinktureinpinselungen behandelt. Nach einem Jahre war noch kcine Besserung eingctreten. Im März I9Io wurde die erste Röntgenuntersuchung vorgenommen. Die Veränderungen veranlaßten den Arzt zur Diagnose Tuberkulose. Neun erfolglose Jodoformöleinspritzungen. Anfangs März I9II begab sich die Patientin in die chirurgische Poliklinik in Basel. Die dort vorgenommene Untersuchung (Dr. I selin) crgab folgendes: Die Haltung und Form der IIand im allgemeinen normal. Keine dilatierten Venen, Sehnen etwas verstrichen. In der Gegend der Carpalknochen ist eine leichte Schwellung zu sehen. Dic Haut darüber ist nicht gerötet und nicht warm. Der Umfang des Handgelenks beträgt links $16,25 \mathrm{~cm}$, rechts $17,25 \mathrm{~cm}$. Der Umfang des Vorderarms oberhalb des Gelenks gemessen ist 17,5 , rechts $16,5 \mathrm{~cm}$. Die Distanz vom Kopf des Metacarpus III zum Processus styloidcus radii ist links 9, rechts $8 \mathrm{~cm}$. Druck genau in der Mitte zwischen beiden; Processus styloidci unmittelbar vor dem Radius schmerzhaft. Zug und StoB in der Längsachse des Gliedes bleibt schmerzlos. Bei aktiven Bewegungen des Handgelenks nimmt man ein fühlbares Krepitieren und Knacken wahr. Es ist aber unmöglich, sich über ein Verschieben von Knochenfragmenten Rechenschaft zu geben.

Funktionsprüfung: Dorsalflexion rechts $135 \mathrm{Grad}$, links I7 I Grad; Volarflexion rechts 135 Grad, links 177 Grad; Ulnaradduktion rechts 30 Grad, links 4 Grad; Radialadduktion rechts 3o Grad, links 4 Grad; Pro- und Supination, Fingerbewegungen normal. Kraft des Faustschlusses mit dem Dynamometer gemessen: Rechts $25 \mathrm{~kg}$ gegen links $15 \mathrm{~kg}$.

Röntgenbild (Fig. Io): MäBiger Grad allgemeiner Osteoporose. Vorderarmknochen normal. Naviculare steil gestellt. Lunatum ungefähr auf die Hälfte seines proximal-distalen Durchmessers verkürzt, wie platt zusammengedrückt, aus dunkler und heller sich zeichnenden Partien bestehend, ohne deutliche Bälkchenzeichnung. Eine völligc Zusammonhangstrennung der einzelnen dasselbe zusammensetzenden Knochenpartien ist nicht sichtbar. Cbriges Handwurzelskelett normal.

Das Allgemcinbefinden ist vorzüglich. Patientin hat keine Zeichen einer aktiven oder überstandenen Lungentuberkulose, in ihrer Familie ist diese Krankheit ganz unbekannt. Sic war auch sonst nie krank und hatte auch keinen andern Unfall erlitten. Da dic Patientin 
sich nicht sofort zur Operation entschlicßen konnte, wurde ein Versuch mit Röntgentherapie zum $Z$ wecke der Beseitigung der Schmerzen vorgeschlagen. Da aber diese Behandlung keinen günstigen Erfolg hatte, wurde in der chirurgischen Klinik in Basel die Operation vorgenommen.

9. V. IgI Operation (Prof. de Qucrvain): BromäthylÄthernarkose. -- Esmarchsche Blutleere. Dorsaler Längsschnitt über der Mitte des Handgelenks. Dic frcigolegte Kapsel ist leicht verdickt und von einer gefblichen Substanz (Jodoform) durchsetzt. Makroskopisch nichts 'Tuberkulose-Verdiichtiges. Das Gelenk wird eröfnet.

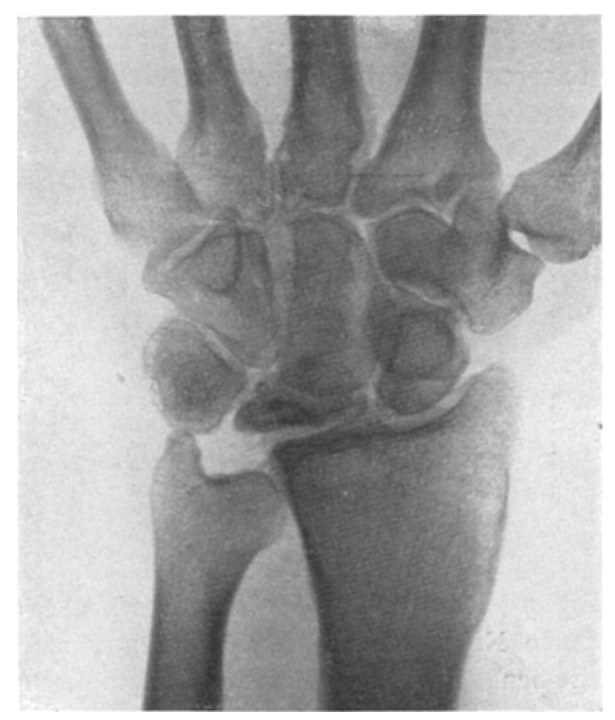

Fig. 1o. Fall 5. Am Lunatum dunkler (nekrotischer) Kern, umgeben von noch ernährtem Knochen.

Das ganze Iunatum erscheint wie zusammengequetscht. Die Gelenkfläche des Lunatum nach dem Naviculare ist gelblich verfärbt und etwas hö̈kerig. Ẍhnliche Veränderungen, aber geringeren Grades, sintl an der anstoßenden Gelenkfläche des Naviculare selbst zu schen. Es wird nun zunächst ein plattes Gebilde aus den lockeren Band. verbindunger ausgelöst, welches die proximo-dorsale Hälfte des Lunatum darstellt. In der Tiefe bleibt ein etwas kleineres gelbliches Knochenstück licgen, das entfernt wird. I)ie übrigen Handwurzelknochen zeigen keine Anomalien. Glasdrain, Naht, Verband in dorsal. flektierter Stcllung.

Heilung p. p. Austritt am I3. V. I9II.

Patientin schrieb seither, daß sie vollständig beschwerdefrei ist 
und daß dic Beweglichkeit des Handgclenks cine bcssere ist als vor der Operation.

Röntgenbild des entfernten Knochens (Fig. II): Proximo-dorsales Stück: Zum Teil unregelmäßig begrenzte Spongiosaschicht von $1 / 2 \mathrm{~cm}$ Dicke mit deutlichem Bälkchengefüge. Daran sich anschließend eine gut ebenso dicke Schicht von Knochen ohne Bälkchenstruktur von etwas unregclmäßigem Gefüge, wenig strahlendurchlässig, nach verkalktem nekrotischen Knochen aussehend. Distalvolares Stück. In die im Röntgenbilde nur als leichter Schatten zur Darstellung kommende fibrös knorpelige Grundmasse sind zwei gröBere und mehrere kleinere Knochenstückchen eingelagert, deren jedes deutliche schwammige Struktur und eine scharfe Grenzlinic aufweist. Nirgends sind zackige Druckflächen vorhanden. Nirgends sieht die Knochenstruktur nach einer tubcrkulösen Zerstörung aus.

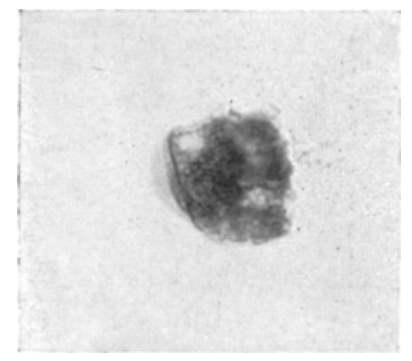

Fig. I a.

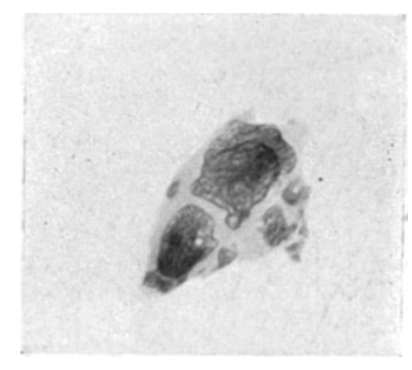

Fig. I I b.

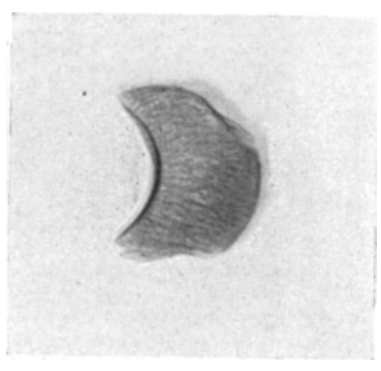

Fig. I2.

Fig. I I a und b. Fall 5. Röntgenbild des Präparates. a) Proximodorsaler Teil des Lunatum. Centrale Nekrose. Peripher lebender Knochen. a) Distal volarer Teil desselben. Fig. I2. Normales Lunatum, zum Vergleich.

Die mit Spongiosa und Kapsel geimpften Meerschweinchen bleiben gesund.

Uber die histologische Untersuchung wird unten im Zusammenhang berichtet werden.

Fa 116 . M. A. (aus der chirurgischen Poliklinik, von Herrn Dr. I s clin beobachtet).

Anamncse: Am 25. VIII. I9I I fühlte der Mann, 32 Jahre alt, beim Heben einer schweren Schiene cin Knacken in der rechten Handwurzel. Von da an hatte er starke Schmerzen in der Hand und war arbeitsunfähig. Er wurde von Dr. M. in P. mit Jodanstrich wegen Tendovaginits crepitans behandelt und dann, am Io. IX., zur Arbeit gewiesen.

Am I8. IX. stellte er sich in der chirurgischen Poliklinik (Dr. Iselin) zur Begutachtung. Es fanden sich die Erscheinungen einer 
Tendovaginitis crepitans der Strecksehnen der rechten Hand, gleichzeitig aber auch cine Schwellung des rechten Handgelenks und eine Druckempfindlichkeit vor dem Radius.

Röntgenbild (Fig. I3 u. I4): Radius und Ulna normal, Handwurzelknochen normal, bis auf das Lunatum. Dasselbe erscheint in seincm proximo-distalen Durchmesser verkürzt, wie abgeplattet und zeigt eine teilweise Verwischung sciner Knochenstruktur. Ganz unregelmäßig geformt erscheint scine dem Triquetrum zugewendete Fläche. Anderweitige Veränderungen im Berciche der Carpalgelenke

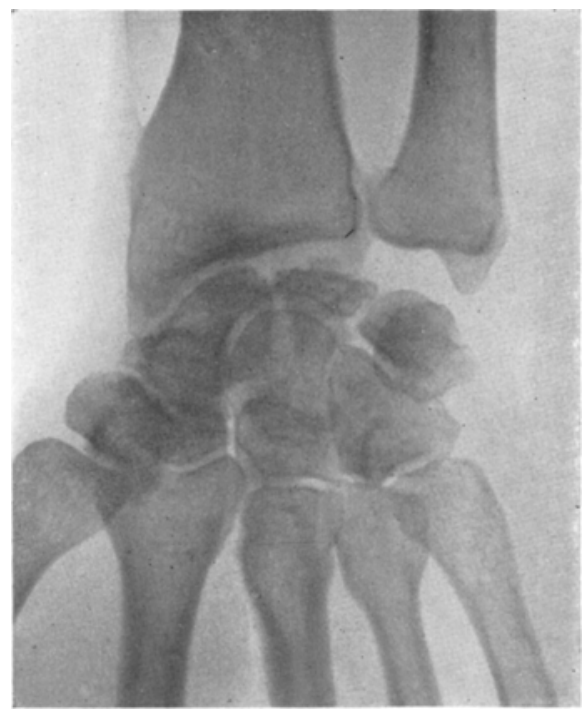

Fig. I 3 .

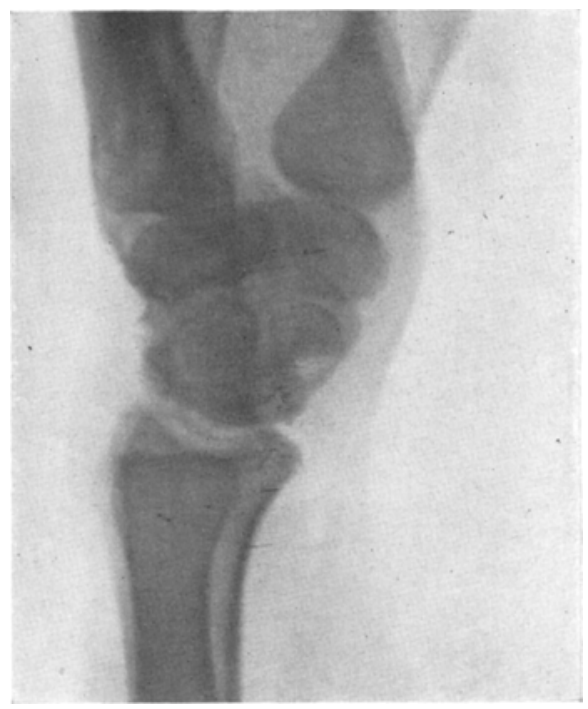

Fig. 14.

Fig. 13. Fall 6. Lunatum stark zusammengedrückt.

Fig. I4. Fall 6. Profilaufnahme. Lunatum volar und dorsal wie „überquellend", stark verkürzt.

sind nicht vorhanden. Es gelingt nicht, den Verletzten zu einer weiteren Nachuntersuchung wieder ausfindig zu machen.

Fa 11 7. 'T. G., Handlanger, 25 Jahre alt, Samaden. (Beobachtet von Herrn Dr. Ruppanner, Spitalarzt daselbst, und Prof. de Quervain.)

Anamnesc: Unfall am 9. VII. I9I2. Patient wollte einen schweren Stein von einer Mauer herunterholen. Dabei fiel ihm dieser Stein in die dorsal flektierte Hohlhand. Sofort starke Schmerzen und Bewegungseinschränkung. Zuerst Behandlung außerhalb des Spitals. Spitaleintritt am 25. VIII. I9I2. 
St t t u s: Sowohl dorsal als auch volar im Bereich der proximalen Handwurzelknochen rechts ausgesprochene Druckempfindlichkeit. Achsendruck nicht schmerzhaft. Starke Bewegungseinschränkung. Perimetermessung crgibt: Dorsalflexion aktiv und passiv I80 Grad, Volarflexion aktiv und passiv r2o Grad, Radialadduktion passiv 30 Grad, Radialaddultion aktiv 20 Grad, Ulnaraddultion passiv $30 \mathrm{Grad}$, Ulnaradduktion aktiv $30 \mathrm{Grad}$.

Röntgenbild (Fig. I5 u. 16): Leichte Osteoporose, Radius und Ulna normal, Naviculare steil gestellt. Lunatum in scinem proxi-

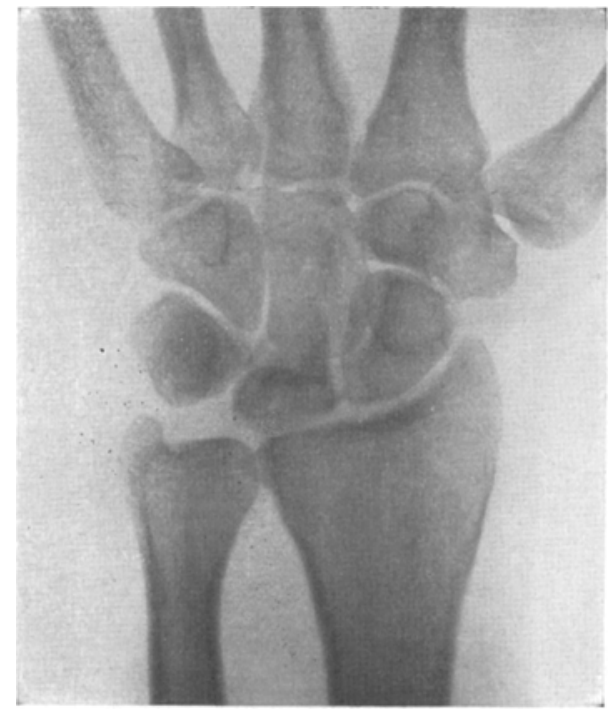

Fig. 15.

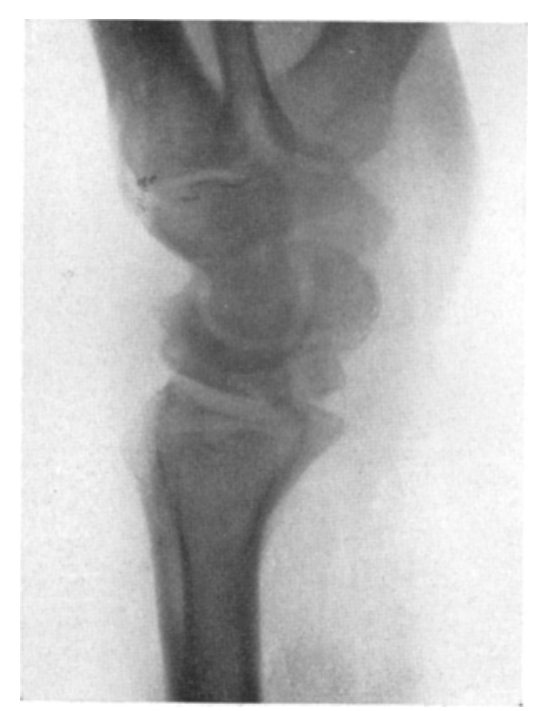

Fig. I6.

Fig. 15. Fall 7. Mäßige Kompression des Lunatum.

Fig. I6. Fall 7. Dorsales und volares ,Überquellen“ des Lunatum.

mal-distalen Durchmesser etwas um $1 / 3$ verkürzt. Knochenstruktur zum Teil unregelmäßig und weniger deutlich als auf der gesunden Seite. Abplattung des Lunatum besonders auf sciner radialen Seite ausgesprochen, da wo es dem Radius anliegt.

Operationsbericht 26. VIII. 1912: Äthertropfnarkose. Dorsaler Längsschnitt ca. $3 \mathrm{~cm}$ lang, etwas radialwärts. Durchtrennung des Lig. carpi dorsale. Eingehen zwischen den Strecksehnen auf das Handgelenk. Eröffnung desselben. Dic Synovialmembrane erscheint etwas gerötet und verdickt, wird zum Teil excidiert und zur histologischen Untersuchung aufgehoben. Das Naviculare zeigt normales Aussehen, soweit es zugänglich ist. Naviculare und Lunatum sind 
in normaler Woise mitcinander verbunden. Das Lunatum zeigt an seinem dorsalen Horn höckeriges Ausschen und ist mit hypertrophischem Knorpel bedeckt. Es fügt sich in die normale Architcktur des Gelenks derart ein, daß auf die Entfernung verzichtet wird. Dageoren wird die höckerige lartic mit dem MeiBel abgetragen. Kapselnaht mit Katgut. Hautnaht.

Der histologische Befund wird später mitgeteilt werden. Spitalaustritt am 2. XI. 1912. Wundverlauf afebril. Glatte I[eilung p. p.

$\mathrm{SchluBstat} u \mathrm{~s}$ : Dorsal über dem rechten Handgelenk eine ca. $5 \mathrm{~cm}$ lange, lineare, verschiebliche und vollständig indolente Narbe. Die Narbe verlïuft nahezu in der Längsachse des Vorderarms und liegt auf der radialen Scite des Handgelenks. In der Gegend des Handgelenks findet sich noch eine ganz minimale Weichteilschwellung. Die Radialinflexion und die dorsale Flexion sind gegenüber der gesunden linken Seite noch in ganz geringem Grade eingeschränkt. I)ie übrigen Bewegungen sind in gleichem Umfange möglich wie links. Die motorische Kraft der linken Hand ist noch etwas herabegesetzt.

Da es sich nicht um einen haftpflichtigen Unfall handelt, fand keine Schätzung eincr eventl. E. U. statt.

Nach Erkundigung beim Arbeitgeber (Ende Dezember 1913) arbeitete der Mann wie früher anstandslos ohne in irgendeiner Weise zu klagen.

\section{Klinisches.}

Nachdem bis I9ro sowohl von anatomischer wie von radiologischer Seite her eine Anzahl von Beobachtungen mitgeteilt worden waren, unter denen wir besonders diejenigen von Pfitzner, GroB, Wittek, Lilienfeld, Blau, Ebcrmayer, Natvig hervorheben, gibt Kienböck IgIo cine cingehende Zusammenstellung des bis dahin Bekannten, und kommt dabei, seine eigenen i6 Fälle eingeschlossen, etwa auf drei Dutzend Beobachtungen, unter denen sich freilich nur ein einziger Operationsbefund vorfindet (GroB), und auch dieser nur mit dem Ergebnis der makroskopischen Untersuchung. Histologische und bakteriologische Untersuchungen fehlten dagegen damals noch völlig oder waren wenigstens noch nicht veröffentlicht worden. Diese Lücke wurde erst während der Niederschrift unserer Arbeit durch eine Mittcilung von Ba um in Kicl ausgefüllt, der an der I4. 'Tagung Nordwestdeutscher Chirurgen kurz über zwei Operationsbefunde mit histologischer Untersuchung berichtet. Boi der glcichen Gelegenheit teilten Lchmann, Oehlecker, zur Verth weitere klinische Beobachtungen mit. 
Wir werden nun im folgenden auf Grund dieses gesamten Beobachtungsmaterials und unterer besonderer Berücksichtigung der uns von Herrn Prof. de Q u ervain zur Verfügung gestellten Fälle das Wichtigste über die isolierten traumatischen Schädigungen des Lunatum hervorheben.

$$
\text { I. Ätiologie. }
$$

Unter den bisher beschriebenen Fällen geben nur 4 ausdrücklich kein Trauma als Ursache der Handgelenkerkrankung an ( 3 Fälle von Kienböck, I Fall von Ebermayer). I Fall Ebermayer schreibt seine Erkrankung dem Hobeln zu, ohne anderweitige Ursache.

Nur unbestimmte Angaben über die Natur des Trauma finden wir ferner bei Fällen von Hirsch (Kontusion der Hand), Oberst (Trauma?), Natvig (Verstauchung der Hand), Kienböck (schwerer Unfall, Kontusion der Hand, Fall vom Dach, Zerrung, Sturz von der Leiter, Sturz vom Wagen), Ba um (Sturz auf die Hand, Quetschung der Hand). Eigener Fall I (heftige Bewegung), Fall 4 (Distorsion). Bestimmter angegeben werden folgende Traumen: Heben einer schweren Schiene, Heben eines schweren Schaftes (Kienböck), Anprallen einer Spitzhacke gegen die rechte Handwurzelgegend (Hirsch), Anprallen eines langen Messerstiels gegen die rechte Hand (Grashey), Drehen eines fest zugemachten Schraubendeckels mit dem dorsal flektierten und zugleich radialwärts gerichteten Handgelenk (diese Arbeit Fall 5), beim Griff „Gewehr über" (Blau), „Nach dem Exerzieren mit dem Gewehr" (K i enböck), Fallen eines schweren Steines auf die Palmarfläche der dorsalflektierten Hand beim fixierten Ellenbogen (Hirsch), Anprallen eines schweren Steines auf die dorsal flektierte Hohlhand (eigener Fall 7), Schlag der Kante eines schweren umkippenden Schrankes (Witteck), Fall auf die dorsalflektierte Hand (diese Arbeit Fall 2), Fallen auf die rechtwinkelig dorsalflektierte Hand (Ebermayer), heftiger Sturz vom Rad auf die überstreckte Hand (Ebermayer), Sturz aus beträchtlicher Höhe mit Auffallen auf die Hände (Groß), Fall auf die gestreckten Hände (Kienböck), heftiges Anprallen der geschlossenen Faust gegen den Boden (Kienböck), Fall auf die rechte Hand bei gebeugtem Handgelenk (Kienböck). 
Die in den anamnestischen Daten über die Entstehung der Verletzung angegebenen Traumen erweisen sich also als sehr verschiedenartig. Allerdings sind die großen Gewalteinwirkungen reichlich vertreten. Es kommen aber auch einfache Kontusionen und Distorsionen, ja sogar unbekannte, besser gesagt unbeachtete Traumen vor, bei denen die Gewalteinwirkung sich auf eine plötzliche hochgradige Muskelanstrengung beschränkte. Dienstmädchen, Taglöhner, Handarbeiter sind tagtäglich bei der Arbeit solchen Traumen ausgesetzt, die ihnen gar nicht als solche zur Wahrnehmung kommen. Es liegt deshalb die Annahme sehr nahe, daß auch den 4 Fällen, bei denen überhaupt kein Unfall angegeben wird, ein solcher indirekter traumatischer Ursprung zugrunde liegt. Immerhin würde in unserer Zusammenstellung bei ungefähr I7 Fällen der „schwere Unfall“ als Ursache der ursprünglichen Schädigung des Mondbeines fehlen. Ein Gemeinsames haben alle die angeführten Schädigungen: In den Fällen, wo Sturz die Verletzung zur Folge hatte, fand sich die Hand ausnahmslos in Dorsalflexionsstellung, öfter wahrscheinlich auch etwas ulnar adduziert. Aus anatomischen Gründen ist es auch sicher, daß der Stoß auf die Vola mehr den Daumenballen und den Radius treffen multe als die Kleinfingerseite. İbenso geht es aus der Natur der Bewegungen hervor, daß beim Heben, Stemmen, Drehen die Hand dorsalflektiert sein mußte, auch wo das nicht besonders angegeben wird. In den Fällen Hirsch (3), Fallen eines schweren Steines auf die dorsalflektierte Hand bei fixiertem Ellenbogen, Wittek, Schlag der Kante eines schweren Schrankes gegen die dorsalflektierte Hand, Grashey-Hirsch, Anprallen des Stieles eines Messers bzw. einer Spitzhacke in der Hand, Bla u und Kien böck, Exerzieren mit dem Gewehr kommt noch der spezielle Umstand in Betracht, daß die Gewalteinwirkung in der Vola eine sehr engbegrenzte war.

\section{Symptomatologie.}

Unmittelbar nach dem Trauma, falls ein solches angegeben worden ist, empfanden die Verunglückten mehr oder weniger heftigen Schmerz. Es scheint, da $\beta$ der Schmerz heftiger und plötzlicher auftrat, wenn die Gewalteinwirkung stärker war, desgleichen wo letztere auf die enger begrenzte Gegend der proxi- 
malen Handwurzelknochenreihe einwirkte. Gleichzeitig wurde bisweilen auch cin Knacken wahrgenommen (eigene Fälle I und 3, Blau, Kienböck). Schmerzen als erstes subjektives Symptom geben auch die Patienten an, bei welchen das ursprüngliche Trauma an Bedeutung zurücktrat und die Affektion von vornherein einen mehr chronischen Verlauf zeigte.

Auch bemerkten mit wenigen Ausnahmen (Ebermayer) alle Verunfallten eine Schwellung des Handrückens. Dieselbe trat meistens sofort auf und dauerte verschieden lang.

Als untrüglichstes Symptom der Verletzung wurde allgemein die Funktionsstörung angegeben. Sie äußert sich durch eine mehr oder weniger ausgesprochene Einschränkung der Bewegungen, namentlich der Dorsal- und Volarflexion. In cinem Falle sogar (Ebermayer) wurden die aktiven Bewegungen vollständig aufgehoben, einerseits durch das heftige Schmerzgefühl, andererscits vielleicht durch das mechanische Hindernis stark dislozierter Knochenfragmente.

Diese drei primären Symptome (Schmerz, Schwellung, Funktionsstörung) sind meist auch noch nachweisbar, wenn die Untersuchung erst nach einiger Zeit stattfindet.

Fast immer wird über das Zunehmen der Beschwerden bei jeder Anstrengung geklagt. Abgesehen von der Anschwellung erscheint dic Hand, weder in ihrer allgemeinen Form noch in ihrer Beziehung zu den Vorderarmknochen, wesentlich verändert.

Mehrfach (Wittek, Kienböck) wurde eine deutliche Verkürzung des Carpus, auch bei fehlender Anschwellung des Handrückens, gefunden. In beiden Fällen zeigt das Röntgenbild ein sehr stark breitgedrücktes Lunatum, das sich in eine Vertiefung der Radiusgelenkfläche versenkt hat.

Bei den Fällen unserer Arbeit, wo die Verkürzung der Distanz Metacarpalknochen III und Processus styloideus radii im Radiogramm sehr deutlich ersichtlich ist, erscheint die Hand in der Carpusgegend fürs Auge immerhin nicht merklich kürzer als die gesunde. Es mag vielleicht die vorhandene Schwellung diesc Eigentümlichkeit verdeckt haben. Diese Anschwellung sitzt auf dem Handrücken. Sie ist meist diffus und geht nie über clen Bereich der Handgelenkgegend hinaus. Es liegt ihr anfäng- 
lich die sofort sich bildende Blutung zugrunde, die sich später größtenteils resorbiert. Infolge weiteren Gebrauches der Hand kann sodann durch chronische Reizung dem organisierten Überrest des Blutergusses sich eine Verdickung der angrenzenden Weichteile (Kapsel, Bänder, Sehnenscheiden usw.) beigesellen. Am wenigsten scheint ein intraartikulärer ErguB in Betracht zu kommen. Manchmal ist endlich eine wirkliche Skelettverdickung greifbar, und zwar in Form einer umschriebenen, harten, druckempfindlichen Prominenz nach vorn von der dorsalen Radiuslippe. In cinem Falle Ebcrmayers ist neben dieser dorsalen Prominenz volar noch eine undeutliche Resistenz tastbar. Der Fall $\mathrm{I} 4$ von $\mathrm{Kienböck} \mathrm{bietet} \mathrm{die} \mathrm{Eigentümlichkeit} \mathrm{dar,} \mathrm{daß}$ bei Fehlen irgendeiner Schwellung der Dorsalseite volar in der Gegend des Naviculare eine zirkumskripte knochenharte Prominenz von über Linsengröße zu fühlen ist. Daß bei der Volarflexion sich diese Vorsprünge mit bewegen, manchmal krepiticren und das Gefühl rauhen Knarrens geben, bedarf keiner weiteren Erklärung. Am ehesten findet man bei der Palpation cine abnorme Ausfüllung der normalen leichten Einsenkung nach vorn von der dorsalen Radiuslippe, in der Mitte zwischen beiden Griffelfortsätzen. Dagegen fehlt - und dies ist diagnostisch sehr wichtig - eine ausgesprochene dorsopalmare Verdickung des Carpus, welche für die Luxation des Lunatum und die typische intercarpale Luxationsfraktur (de Quervain$\mathrm{Schoch)}$ so bezeichnend ist. Die Druckempfindlichkeit wird an der Stelle der l'rominenz, wo letztere fehlt, in der LunatumNaviculargegend am stärksten empfunden. In einem einzigen Falle wird bemerkt, daß der lokale Druck keinen Schmerz erzeugt hätte. Wo er geprüft wurde, löste der StoB an der Hand in der Richtung der Vorderarmachse Schmerz aus (diese Arbeit Fall I-3). Fine Fernwirkung des 1)ruckes, Schmerz in der Lunatumgegend bei Druck auf das Capitulum metacarpi III sieht man in einem Falle Kienböcks; Zug an der Hand schmerzt nicht (diese Arbeit Fall 2 und 5).

Boi der wenigen augenfälligen Eigenart unserer Verletzung läßt doch die Funktionsprüfung fast in allen Fällen beträchtliche Störungen der Beweglichkeit erkennen. Vollständiges Aufgehobensein der aktiven Bewegungen trafen wir zwar nur einmal 
(Ebermayer, siehe unter Prognose). Im Gegensatz zu dem steht ein anderer Fall von Eberma yer, in welchem nur geringe Störungen auftraten (Beugebehinderung von etwa I5 Grad), sowie unser Fall 7. Zwischen diesen beiden Extremen steht die Mehrzahl unserer Verunglückten, bei denen immerhin die Ausübung der Berufsarbeit für längere Zeit unmöglich gemacht wurde.

Leider geben nur sehr wenige Autoren genaue Messungsresultate der Gelenkfunktion. Vielfach wird angegeben, daß die passiven Bewegungen ausgiebiger, aber schmerzhafter sind als die aktiven. So viel ist jedenfalls ersichtlich, daß bei unserer Verletzung wahrscheinlich keine typische Funktionsstörung auftritt, im Gegensatz zu der Lunatumluxation und der intercarpalen Luxationsfraktur, bei denen sich primär oder sekundär Störungen der Fingerbewegungen bemerklich machen. In einigen Fällen ist die Volarflexion des Handgelenks stärker gehindert als die Dorsalflexion. In anderen wiederum wird das Umgekehrte getroffen. Die Ab- und Adduktionsbewegungen können beinahe ganz aufgehoben sein (diese Arbeit Fall 5). Meistens aber sind sie wenigstens stark eingeschränkt, selten wurden sie normal gefunden.

Über die Pro- und Supination finden sich nur selten Angaben vor. In unseren Fällen 2 und 5 war sie normal. $\mathrm{Ob}$ in frischen Fällen eine seitliche falsche Beweglichkeit vorhanden ist, wird nirgends angegeben, ist aber von vornherein sehr unwahrscheinlich.

Über die Kraft des Faustschlusses werden nur 5 mal Angaben gemacht. Sie ist gut in einem Fall von Ebermayer, wo die aktiven Bewegungen noch relativ gut ausführbar sind, dagegen fand ihn deQuervain um die Hälfte (Fall 3) und mehr (Fall 2 und 5) vermindert bei merklicher Bewegungshinderung im Handgelenk.

Es ist leicht verständlich, daß durch den Nichtgebrauch der Hand mehr oder weniger ausgesprochene Muskeltrophie entstehen kann, wie sie in einigen Fällen getroffen wurde.

Da wir uns streng an die Besprechung des is olierten Mondbeinbruches halten, gehen wir hier auf die durch Komplikationen hervorgerufenen Symptome nicht näher ein. 
Es sei einzig bemerkt, daß in einem Falle ( $\mathrm{K}$ ienböck) bei der Lunatumquetschung über, auch in der Nacht anfallsweise auftretende Schmerzen, die vom Vorderarm, zuweilen auch in die Finger ausstrahlen, berichtet wird. Derartige Schmerzen, allerdings streng auf das Medianusgebiet beschränkt, sind viel häufiger bei den Luxationen des Lunatum und den Luxationsfrakturen, wo das verschobene Knochenstück auf den Stamm des Nervus medianus drückt.

Von besonderer diagnostischer Bedeutung ist die Tatsache, daß in den Fällen, welche längere Zeit hindurch beobachtet wurden, die Erscheinungen sich nach intensiverer Inanspruchnahme des Gelenks durch schwerere Arbeit häufig steigerten, und daß ab und zu nach plötzlichen Anstrengungen hochgradigere Störungen einsetzten, dic dann allmählich wieder zurückgingen.

Fassen wir das eben Gesagte zusammen, so sehen wir, daß der Verletzte anfänglich die gewöhnlichen Erscheinungen einer Handgelenksdistorsion aufweist, daß dieselben aber nicht in gewohnter Weise zurückgehen, sondern einem chronischen, bei stärkerer Beanspruchung des Gelenks exacerbierendem Zustande Platz machen, der in den meisten Fällen gekennzeichnet ist durch eine Einschränkung der Handgelenksbewegung, eine leichte Druckempfindlichkeit nach vorn von der dorsalen Radiuslippe und bisweilen eine leichte Schwellung daselbst. Dabei sind die extremen Gelenksbewegungen schmerzhaft, und es bestehen öfters auch spontane Schmerzempfindungen. Über die diagnostische Bedeutung dieser Zeichen werden wir weiter unten sprechen.

\section{Röntgenbefunde.}

Line reichhaltige Zusammenstellung der verschiedenen Typen von Röntgenbefunden finden wir in der oben erwähnten grundlegenden Arbeit von Kienböck. Wir begnügen uns deshalb hier die wichtigsten Veränderungen kurz hervorzuheben, besonders mit Rücksicht auf unsere eigenen Befunde.

Was zunächst clie allgemeine Gelenkkonfiguration des Carpus anbelangt, fällt meist eine deutliche Verkürzung desselben in die Augen. Sie rührt davon her, daß das Lunatum in der Längsrichtung des Gliedes kürzer ist als das der gesunden Seite (alle Fälle unserer Arbeit, Ebermayer, Hirsch, Wittek, Kien- 
böck). Je auffallender die Verkürzung des Lunatum ist, um so mehr sehen wir auch, wie aus unseren eigenen Bildern hervorgeht, das Naviculare steilgestellt.

Neben diesen Verhältnissen zeigen andere Aufnahmen noch eine förmliche Versenkung des betroffenen Mondbeines in unserem I. Falle nach I2 Jahren des Naviculare in die vertiefte, ausgehöhlte Gelenkfläche des Radius (Kienböck, Wittek).

Außerdem sehen wir in einigen Fällen Kienböcks Verschiebungen des deformierten, verkleinerten Lunatum. In seinen Fällen I I, I2, I 5 erscheint das Mondbein um das Capitatum dorsalwärts verschoben, bei ,Fall $\mathrm{I}_{4}$ und $\mathrm{I}_{5}$ ist das Lunatum durch Abschrägung der proximalen Fläche, namentlich nach der navicularen Seite, gedreht, seine distale Gelenkfläche sieht ein wenig navicularwärts, und so ist das Capitatum samt dem Hamatum daumenwärts abgeglitten.

Am Lunatum selbst sind Veränderungen sowohl in der Form als in der dorsalen Struktur zu erkennen. Der proximale Teil des Knochens scheint fast ausnahmslos am stärksten betroffen zu sein. Man trifft ihn keilförmig zusammengedrückt (E bermayer), abgeflacht (Ebermayer), dabei noch durch Resorptionsvorgänge im Sinne einer deformicrenden Ostitis (eigene Fälle) stark verändert. Andermal fehlt der proximale Teil. An seiner Stelle finden sich cinzelne Knochensplitter (Natvig, $\mathrm{Hirsch}$ ). Bisweilen finden sich auch abgesprengte Stücke meist an der proximo-ulnaren Ecke (Ebermayer, Wittek). Sehr selten scheint der Befund zu sein, bei welchem das Lunatum deutlich in zwei bzw. mehrere Stücke zerfallen ist. In einem Falle Ebermayers ist die Zersprengung in zwei Stücke besonders schön zu erkennen dadurch, daß die Fragmente stark dorsalwärts und volarwärts voneinander disloziert wurden. Ganz besonders schön ist die Auflösung des Lunatum in zahlreiche größere und kleinere Bruchstücke im Röntgenbilde zu sehen, welches von unserem vierten Falle nach der Operation angefertigt wurde. Wir werden uns über die Deutung desselben später aussprechen. Hier sei noch ein Fall erwähnt, der während der Niederschrift dieser Arbeit in der chirurgischen Klinik in Basel zur Beobachtung kam und der uns von Prof. de Querva in noch mitgeteilt wurde. Nach einem Sturz auf die dorsalflektierte 
Hand fand sich in der Profilaufnahme der Kopf des Capitatum volarwärts verschoben und das volare Horn des Lunatum abgerissen und ebenfalls volar und proximal verlagert. Wir werden auf diesen Fall bei der Besprechung des Mechanismus noch eingehen.

Jie Strukturveränclerungen äußern sich einmal durch Unregelmäßiơwerden und Schwinden der feinen Bälkchenstruktur des Knochens und sodann durch unregelmäßige und ungleiche Verteilung des Kalkes im Knochen. Es zeigt sich im dorsovolaren Bilde bald eine schmale Zone, bald ein zentraler rundlicher Teil des Lunatum auffallend undurchsichtig, kalkhaltig, während der übrige Knochen ungewöhnlich stark aufgehellt ist. In einzelnen Iällen ist die aufgehellte Zone wieder von einer scharfen dunkler gezeichneten schmalen Knochenschale umgeben.

In unseren Fällen I (erstes Bild), 4, 7 sind die Veränderungen verhältnismäßig wenig weit gediehen und betreffen besonders den proximalen Anteil des Knochens, der nach dem Radius hin eine einigermaßen gewellte Grenzlinie erhält. Ausgesprochener ist im dorsopalmaren. Bilde die Abplattung im Falle 3 und 6, und noch hochgradiger im Falle I (zweite Aufnahme) und 5. Hier macht das Lunatum vollständig den Eindruck, flachgedrückt zu sein.

Ganz verschicden hiervon ist der Befund im Falle 2. Hier finden wir in schönster Ausbildung, ähnlich wie die Fig. 8 der Tafel zur Kienböckschen Arbeit, die zentrale Partie des Lunatum für Röntgenstrahlen sehr undurchlässig, hochgradig verkalkt, in einer dünnen, zum Teil unterbrochenen Knochenschale liegend.

In der sagrittalen Aufnahme erscheint das Lunatum meist in seincr proximo-distalen Richtung verkürzt, an seinen beiden Gelenksflächen abgeflacht, dorsal, und besonders volarwärts abnorm stark vorspringend, ja in ein dorsales und ein palmares Stück geteilt (Fall I und 5).

Was endlich die Veränderung der Nachbarknochen betrifft, so findet sich bcinahe immer ein gewisser Grad von Osteoporose vor. Anderweitige Veränderungen haben wir in unseren frischen Fällen nicht gesehon. In dem über I I Jahre beobachteten Falle I unserer Arbeit fanden sich dagegen bei der späteren Aufnahme (Fig. 2, 3) deformierende Veränderungen an allen das Radio- 
carpalgelenk betreffenden Knochen ausgebildet, freilich in viel geringerem Grade als an dem primär erkrankten Lunatum. Der Radius zeigt an seiner Gelenkfläche eine Vertiefung, in welche das Naviculare eingelagert ist. Seine ganze Gelenkfläche ist überdies etwas unregelmäßig und ebenso die Bälkchenstruktur der Epiphyse und der Metaphyse. An der Ulnae ist der Processus styloideus in seiner Form verändert. Leichtere Unregelmäßig. keiten zeigen die Gelenkflächen des Triquetrum und des Naviculare. Von den Knochen der zweiten Reihe zeigt nur der Kopf des Capitatum eine etwas unregelmäßige Begrenzung. Die übrigen Knochen sind normal, ebenso wie die Metacarpalknochen.

Auch im Falle 2 zeigt die Nachuntersuchung - 9 Jahre nach der Entfernung des Lunatum - an den die Lunatumlücke begrenzenden Knochenflächen deutliche deformierende Veränderungen, wenn auch etwas weniger ausgesprochen als Fall I, bei dem das Lunatum geblieben ist.

Ferner sei an die Beobachtungen von Wittek, Grashey, Kienböck erinnert, in denen die Gelenkfläche des Radius unregelmäßig vertieft, sozusagen ausgehöhlt erschien, so daß sich das veränderte Lunatum mehr oder weniger in eine Stelle des Radius einlagerte.

\section{Diagnose.}

Dieselbe wurde in keinem unserer Fälle vor der Aufnahme des Röntgenbildes gestellt, und allem Anscheine nach bei den in der Literatur mitgeteilten Fällen ebensowenig. Wie wir aus der Symptomatologie ersehen haben, bietet sie ja eigentlich keine auf den ersten Blick kennzeichnende Symptome dar.

Für die Differentialdiagnose müssen wir die frische Verletzung und die Spätfolgen derselben auseinanderhalten. Wir tun dies aber mit dem Vorbehalte, daß wir über das $\mathrm{frische}$ Bild ganz besonders in bezug auf die Röntgenveränderungen eigentlich sehr wenig wissen. Die ersten Erscheinungen nach der Verletzung wurden gewöhnlich entweder dem Arzte gar nicht gezeigt oder von diesem als einfache Distorsion aufgefaßt und demnach nicht mit Röntgenstrahlen untersucht. Erst wenn die Beschwerden nicht weichen wollten oder sich gar in der Folge steigerten, wurde zu diesem Untersuchungsmittel gegriffen, und 
wurden dann Veränderungen gefunden, denen man es auf den ersten Blick ansehen kann, daß sie nicht den Charakter einer frischen Knochenverletzung tragen. Wir können also das frische Verletzungsbild eigentlich bis jetzt nur retrospektiv aus den in der Anamnesc erhältlichen Angaben zusammenstellen, und da unterscheidct es sich eben nicht von dem Bilde einer gewöhnlichen Handgelenksdistorsion. Höchstens könnte darauf aufmerksam gemacht werden, daß der Hauptschmerz sich nach vorn vom dorsalen Rande des Radiusendes lokalisiert hat, also im Bereich des Mondbeines. Dies ist aber so oft auch bei einer gewöhnlichen Distorsion der Fall, daß ein sicheres differentialdiagnostisches Merkmal auch hierin nicht zu finden ist. Etwas bezeichnender wäre vielleicht Achsendruckschmerz, besonders bei Stoß in der Richtung des dritten Metacarpus (F insterer). Bei schwerer Kertrümmerung des Lunatum wäre wohl auch eine Ausfüllung der leichten Depression vor der Radiuslippe zu finden und vielleicht eine geringe Verkürzung der Hand vom Köpfchen des dritten Metacarpus zum dorsalen Rande der Radiusgelenkfläche. Jicses von Finsterer angegebene Zeichen ist wohl für die Spätveränderungen bezeichnend, nicht aber für die frische Verletzung:

Leichter als der Distorsion gegenüber ist die Differentialdiagnose zwischen frischer Lunatumverletzung und den übrigen klassischen Verletzungendes Handgelenks, der Frakturdes Naviculare, der Luxation des Lunatum und der intercarpalen Luxationsfraktur. Da von den gebräuchlichen Lehrbüchern nur die Diagnostik von de Quervain auf diesen Punkt genauer eingeht, so seien die wichtigsten Kennzeichen hier kurz hervorgehoben:

Der gewöhnlichen Navicularefraktur gegenüber bezeichnend ist für die Quetschung des Lunatum nur der mohr ulnare Sitz des lokalen Druckschmerzes. Beim Kahnbcinbruch findet sich derselbe mehr in der Tabatière. Wo diese Schmerzlokalisation nicht ausgesprochen ist, da ist auch eine Differentialdiagnose unmöglich.

Von der gewöhnlichen volaren Luxation des Lunatum unterscheidet sich unsere Verletzung durch das Fehlen der bei Luxation stets deutlichen dorso-volaren Verdickung des Hand- 
gelenks bzw. durch das Fehlen des deutlichen Knochenvor. sprunges unter den Beugesehnen. Auch fehlen die bei der Lunatumluxation so häufigen Funktionsstörungen von seiten der Beugesehnen und die Kompressionserscheinungen von seiten des Nervus medianus. Die sehr seltene dorsale Luxation des Lunatum läßt sich an dem dorsalen Knochenvorsprunge erkennen.

In gleicher Weise wie von der volaren Luxation des Lunatum unterscheidet sich unsere Verletzung auch von der intercar. palen Luxationsfraktur, d. h. von der volaren Luxation des Lunatum mitsamt der proximalen Hälfte des quergebrochenen Naviculare. Bei dieser Verletzung ist die dorso-volare Verdickung des Handgelenks eine noch auffallendere als bei der reinen Lunatumluxation, und eine Verwechshung dürfte also bei genauer Untersuchung nicht vorkommen.

Von der Radiusfraktur unterscheidet sich unsere Verletzung durch die Beschränkung des Druckschmerzes auf die Gegend vor dem Radius. Eine Verwechslung könnte nur vorkommen mit jenen seltenen Fällen von $A b r i B$ der dorsalen Radiuslippe. Dieser Verletzung gegenüber wird allerdings nur das Röntgenbild sicheren Aufschluß geben.

Für die Differentialdiagnose der chronisch gewordenen Lunatumschädigung kommen einmal wieder die Spätfolgen der eben genannten Handgelenksverletzungen: also der Navicularefraktur, der Luxation des Lunatum, der intercarpalen Luxationsfraktur und des Abbruches der dorsalen Radiuslippe in Frage. Die differentialdiagnostischen Momente und Anhaltspunkte wcrden wieder dieselben sein, von den akuten Äußerungen der Verletzung abgeschen. Den Ausschlag wird auch wieder hier das Röntgenbild geben.

Vom praktischen Standpunkte aus nicht minder wichtig ist aber die Differentialdiagnose des chronisch gewordenen Zustandes gegenüber chronischen Entzündungsprozessen am Handgelenk und seiner Umgebung. An eine Sehnen- bzw. Sehnenscheidenerkrankung wird man kaum denken, wenn nicht, wie zufällig in unserem 5. Falle, gleichzeitig eine krepitierende Tendinitis und Peritendinitis besteht (vgl. auch eine Beobachtung von Ebermayer).

Wichtiger ist die Unterscheidung von Tuberkulose und 
cluronischer Arthritis deformans. 6 mal, einschließlich von 4 unserer eigenen Fälle, wurde an Tuberkulose gedacht. Im I. Falle unserer Zusammenstellung hatte Prof. de Quervain in Ermangelung anderer Anhaltspunkte die Diagnose mit Wahrscheinlichkeit auf Tuberkulose gestellt und die Patientin dementsprechend mit Jodoformininjektionen behandelt. Erst auf Grund der Vergleichung des Röntgenbildes mit den späterhin von ihm beobachteten Fällen kam er zur richtigen Diagnose einer traumatischen Schädigung des Lunatum. In unserem 2. Falle besonders gewann diese Frage deshalb eine größere Bedeutung, weil es sich um einen gegen Unfall versicherten Patienten handelte. Vom Arzte der Versicherungsgesellschaft wurde dic Diagnose denn auch wirklich auf Tuberkulose gestellt, und erst die histologische und bakteriologische Untersuchung der bei der Operation gewonnenen Gelenksteile sicherte dem Patienten sein volles Recht auf Entschädigung. Auch im Falle 4 wurde an Tuberkulose gedacht, da das Trauma nicht erwiesen schien. Weitere Beobachtung hätte den Fall aufgeklärt, doch entzog er sich derselben, da die Symptome schwanden. Ebenso wurde in unserem 5. Falle von dem erstbehandelnden Arzte auf Cirund des Röntgenbildes eine Tuberkulose angenommen und wurde dic Patientin dementsprechend, allerdings völlig erfolglos, mit Jodoforminjektionen behandelt.

Schon der klinische Verlauf erlaubte meist nach einigen Monaten, jeden Zweifel auszuschließen. Während dic Veränderungen bei unserer Lunatumläsion sich stets im engen Rahmen der Umgebung dieses Knochens abspielen, greift die tuberkulöse Erkrankung meist allmählich auf das übrige Handgelenk über und fülırt zum mindesten zu einer stärkeren Weichteilschwellung und zu einer hoheren örtlichen Temperatursteigerung als dies bei unserer Verletzung der Fall ist, und ganz besonders zu einer bei der traumatischen Lunatumläsion fehlenden Störung der Fingerbewegung. Für die traumatische Lunatumläsion bezeichnend ist gerade die Hartnäckigkeit der an sich scheinbar geringfügig bleibenden Veränderungen und das lange Bestehenbleiben eincr Funkitonsstörung im Handgelenke, trotzdem die äulberlich greifbaren Erscheinungen ciner Tuberkulose fchlen und die Beweglichkeit der Finger eine normale bleibt. Noch rascher 
und sicherer entscheidet aber das Röntgenbild. Während man in den ersten Zeiten, wic dies in 3 unserer Fälle geschehen ist, auch bei Betrachtung der Röntgenplatte noch zuerst an Tuberkulose dachte, so wird der Erfahrene in Zukunft auf Grund des Röntgenbildes ohne weiteres die richtige Diagnose stellen, wenn schor die Lunatumschädigung in gewissen Fällen, besonders in denjenigen mit scheinbarer Sequestrierung des Zentrums des Knochens, einer tuberkulösen Veränderung ähnlich sicht. Bezcichnend ist auch hier wieder die Beschränkung der Knochenveränderungen auf das Lunatum und das Intaktbleiben der umgebenden Handwurzelknochen bis auf nach Jahren sich ausbildende Erscheinungen deformierender Ostitis. Bei Tuberkulose schen wir, daß in der Regel auch die Nachbarknochen an dem P'rozesse teilnehmen, so dal3 es schließlich zu einer wolkigen Aufhollung des ganzen Carpalskeletts kommt.

Auch von der nicht tuberkulösen chronischen deformierenden Arthritis unterscheidet sich unsere Verletzung dadurch, daß sie auf einen cinzigen Knochen bzw. auf seine unmittelbare Umgebung beschränkt bleibt, während die klassische deformierende Arthritis zum mindesten die beiden Handgelenke symmetrisch befällt, und auch hier stets zur Veränderung von mehreren, ja von allen Knochen zugleich führt.

Verwechslung mit anderweitigen entzündlichen Gelenkcrkrankungen, so mit gonorrhöischer Arthritis dürfte wohl kaum vorkommen und wird durch die Anamnese und durch das Röntgenbild ohne weiteres auszuschließen sein.

\section{Pathologische Anatomie.}

Wir verfügen bis jetzt noch über keine anatomischen I3eobachtungen von frischen isolierten Verletzungen des Mondbeines. Dies rührt davon her, daß derartige Fälle weder frisch operiert werden, noch auch frisch zur Autopsie kommen, es sci denn, daß die Mondbeinverletzung nur eine Nebenverletzung anderweitiger tödlicher oder zur Amputation führender traumatischer Einwirkungen darstelle. Frische Lunatumverletzungen in diesem letzteren Sinne finden wir von zwei Autoren beschrieben.

I. F' a 11 von $H$ unt. 25jähriger Mann, war aus dem Fenster des zweiten Stockes 20 Fuß hoch herabgefallen. Wegen ciner Ellenbogen- 
Der Kompressionshonch und lie traumatische Erweichung des Mondbcines. I 47

gelenksverletzung wurde die Amputation gemacht. Fs fand sich bei der Autopsio des Armes cine Fraktur des unteren Fincles des Radius, des Lunatum und des Processus styloideus ulnae vor. IDas Lunatum fand sich quer in seiner Mitte gebrochen. Sein volares liragment war an der entsprechenden Partie des Gekenkendes des Raclius sowie an der Ulua hangen geblieben.

2. Fall rom I'este. I'cste fand bei rinem an ten Folgen eines Schädelbruches gestorbenen Patienten eine I,uxationsitellung in rechten Radiuscarpalgolenk mit Abril3 des Processus styloideus radii und ulnac. Das I unatum war gleichacitig Sitz cines doppelten Bruches. Der Stury hatte aus der Itöhe des vierten Stockwerkes stattgefunden. Jieran schliefen sich, obwohl nicht vollie frisch:

3. Fa ll von Riedel (zitiert im Königschen Lehrbuch der allgemeinen Chirurgie und in der Arbeit von GroB). Ein 4ojahriger Mann hatte vor 4 Wochen durch Umfallen eines schweren Kastens eine starke I)istorsion der Hand erlitten. Es zeigte sich sofort starke Schwellung des Fandgelenks, nach deren Verschwinden auf dem 1)orsum manus cin beweglicher Körper nachgewiesen wurde. Es hat sich bei der Operation ein $2 \mathrm{~mm}$ langes, $43 \mathrm{~mm}$ dickes Knorpelknochenstück von dem unteren dorsalen 'leile des Radius abgesprengt; das Os lunatum war in zwei gleich große Stucke zerschlages, wom Triquetrum fehlte der radiale Teil ganz. Er war wahrsehcinlich nach der Vola verschoben worken.

4. Fa 11 von G o B. Ein z-jähiger Mann war vor 2 Jahren aus beträchtlicher IJöhe auf die Iäinde gestürzt. Starke Schwellung des rehten Handgelenks, die aber zum 'Teil zurückging, sich aber besonders bei Nustrengungen wieder einstellte. Die Operation zeigte das I unatum in drei Teile zersprengt, so daß von cinem gröberen leil con kleiner getrennt und dieser wieder in ewei fragmente gretrilt ist.

Daran schlieBen sich jene von I'fitzner zusammengestellten Anomalien des Mondbeines, welche von ihm zum Teil als kongenital aufgefalist und mit dem Namen Epilunatum und Hypolunatum bezeiclnet worden sind, welche aber in Wirklichkeit $A b$ ribfrakturen der beiden Höner des Lunatum darstellen dürften, und ferner die folgenden für uns wichtigeren Beobachtungen Pfitzners:

IV. Beitras, Iland $\mathrm{Nr}$. I3I, rechte Hand cines 54jahrigen Manne's. ,Das Lunatum war in zwei annähernd gleich große Stücke, ein dorsales und ein volares, zerfallen, dic sich mit eburnierten Schlifeflichen berihrten." Alle Carpalia zeigten mannigfache Exostosen lind Abschleifungen, das Carpo-Metacarpalgetenk cles I)aumens war infolge soleher Prozesse vollständig volar gelagert. Ferner bestand 
cine mit massigem Callus geheilte Fraktur des Mctacarpale V. Auch in der linken Iland des Mannes zeigten Grundphalanx II und II! schief verlaufende, schlecht geheilte Frakturen.

V. Bcitrag, Hand Nr. I2, ,rechtseitiges Lunatum cines 3 I jährigen Mannes": Die distale und radiale und ulnare Gelenkfläche sind normal. An der proximalen Fläche ist der Knorpel sanz zerfetzt, in ihm steckten cin größeres und klcinere Knochenfragmente. Auf dem Radius ist dic Faccte für clas Naviculare normal, die für das Lunatum zeigte Knorpelusur und Knochenschliff. Sonstige etwa traumatische Veränderungen bestanden nicht.

VI. Beitrag, $\mathrm{Nr}$. I15, linke IIand eines 55jührigen Mannes: Lunatum in der Woise zerfallen, daß die distale Hïlfte mit der Gelenkfläcle für das Capitatum ziemlich gut crhalten war, und das größte liragment bildete, während die proximak filäche aus mehreren ungestalteten Stücken bestand, die cinzelnen Fragmente waren durch Bandmassen ziemlich locker verbunden, das Verhalten des Radius konnte nicht festgestellt werden. Sonst keine Andcutung von Verletzungen oder entzündlichen Prozessen. Rechte Hand normal.

VIII. Beitrag. Nr. I360, rechte Hand eines 5 ljïhrigen Mannes: Lunatum pathologisch in mehrere Stïcke zcrfallen, dic ihm zugekchrten Flächen des Capitatum und Radius zeigen ebenfalls Knochenschliff.

Os hamuli selbständig, klein, $7 \mathrm{~mm}$ großer Durchmesser, gleitet auf cincm halblangen Stumpf. I.inke Hand normal.

IX. Beitrag, Nr. I388, rechte Hand eines 56jährigen Mannes: Lunatum in zwei große und mehrere kleine Stücke zerfallen. Die urspründliche distale, radiale und ulnare Gelenkfläche sind fast intakt. I) proximale dagegen ist ganz zerstört, die distale Gelenkfläche des Radius ist gan\% intakt. - Sonstige etwa traumatische Veränclerungen bestanden nicht.

Als erste in der Literatur mitgeteilt histologische Untersuchungsbefunde müssen wir hier diejenigen von Baum anschließen. Derselbe fand, daß es sich in seinen beiden Fällen um Fraktur mit ausgedehnter Zcrmalmung und Nekrotisierung der Spongiosa handelte. Die Folge ist nach meiner Auffassung einmal teils Narbenbildung, teils proliferierende Prozesse zur Bescitigung der Nekrosen, vor allem cine starke, wenn auch verzögerte Callusbildung. Der noch weiche Callus ist starken mechanischen Impulsen von seiten des Radius ausgesetzt, was neue Frakturen, dissezicrende und arthritische Prozesse zur Folge hat. Für einc Ostitis fibrosa cystica liegen nach $\mathrm{B}$ a u m in seinen Fällen keinerlei Anhaltspunkte vor, Er bezeichnet das Krankheitsbild 
als durch cine pseudarthritische bzw. verzögerte Heilung der Nondbeinfraktur mit anschließender Arthritis des Radialgelenks. Was bei Bat um fehlt, das sind Angaben über die Verteilung der Veränderungen auf die verschicelenen Teile des Lunatum, Lagre der nekrotischen Partien usw.

Eigene lialle. Wir beginnen mit dem makroskopischen Befunde: Im Falle 2 lösten sich die dorsalen $2 / 3$ des Knochens, bestehend aus dem osteosklerotischen Kern und seiner dorsalen Hülle, von dem volaren Drittel beim Herausheben ab. Der Zusammenhang der beicken Teile muß ein wenig fester gewesen sein, da keine besondere Gewalt angewendet wurde. $\Lambda \mathrm{n}$ dererscits handelte es sich aber doch nicht um zwei Iragmente mit deutlichen Bruchflächen, sondern der Zusammenhang der die zertrümmert aussehenden Spongiosa auf der volaren Seite mit dem osteo-sklerotischen Kern verband, war bloß ein weniger fester als derjenige zwischen der volaren Knochenschale und den volaren Bandmassen. Das makroskopisch Charakteristische an dem Falle war also die sklerotische Umwandlung des zentralen Teiles des Lunatum und seine relative Ablosung von der ihm umgebenden dünnen Knochenschale, die dem Kern an verschicdenen Stellen verschieden fest anhaftete.

Im Falle 5 fand sich das I unatum zusammengedrürkt und in zwei größere Fragmente geteilt, von denen das dorsale eine noch vaskularisierte periphere Schicht und einen nokrotischen stark verkalkten zentralen Teil aufwies. Das volare Fragment enthielt, durch fibröses Bindegewebe vereinigt, zwei größßere und mohrere kleinere Knochenteile, alle aus schwammig ausschender Spongiosa bestehend, ohne die parallele Bälkchenstruktur des normalen Lunatum.

Im Falle 7 stellte sich das Lunatum, wie schon aus dem Röntgenbilde wahrscheinlich greworden war, als zusammenhängendes Ganzes dar, dessen von der Dorsalseite zugängliche Partic allerdings höckerig verändert war, das sich aber doch dem Handgelenk noch so sehr als Ganzes einfügte, daß von seiner Entfernung Alsstand genommen wurde. Eine höckerige Partie muß dem Röntgenbilde nach auch auf der Volarseite vorhanden gewesen sein, doch schien dieser Umstand zu ciner Excision des Knochens nicht zu berechtigen. 
In unseren übrigen Fällen bleiben wir in unseren Schlüssen auf den klinischen Befund und das Röntgenbild beschränkt, da dieselben nicht opericrt wurden.

Wir kommen zu unseren histologischen Befunden:

Dic Untersuchung von Fall 2 war I 904 von Herrn Professor de Quervain vorgenommen worden, diejenigen von Fall 5 und 7 wurden von Herm I'rofessor Dr. Hedinger, Vorsteher des patholog. Instituts der Universität Basel, ausgeführt, der die Güte hatte, mit uns auch die Präparate von Fall 2 noch einmal durchzusehen.

Befund ron Fall 2: Im Zentrum des Knochenstickchens sicht man eine ausgedehnte Nekrose, dic sowohl die Knochenbälkchen, als das Knochenmark betrifft. Die Knochenbälkchen lassen norh lamellären Aufbau erkennen. Eine Kernfarbung der Knochenzellen ist dagexgen nirgends melır möglich. An ciner Stelle greift die Nekrose in geringer Ausdehnung auf den Knorjel iiber. Nach außen von der nekrotischen Partie folgt zellreiches Bindegewebe, das aus kleinen Spindelzcllen zusammengesetzt ist, und in welchem diffus zerstreut reichliche Lymphocyten liegen. Dicse zellreiche Zone wird nach außen von einer etwas zcllärmeren Bindegewebslapsel abgelöst, in der meist schmale Knochenbälkchen liegen, die fast durchweg einen schmalen Saum von Ostcoid reigen, auf dem nach außen hin cine kontinuierliche Reihe von Osteoblasten folgt. Seltener sind kleine Osteoblasten außen. Wicder an anderen Knochenbälkchen zeigen sich neben den Osteoblastensäumen an anderen Stcllen Jlowshipsche Jakunen mit reichlich Ostcoklasten, dic zum Teil mit Blutpigment beladen sind.

F all 5. Das größere, proximo-dorsale Stïck zeigt folgenden histologischen Befund: An cincr Stelle findet sich im Zentrum des Schnittes eine ziemlich ausgedehnte Nckrose, welche dic Knochenbälkchen betrifft. Um diese Nekrose herum sicht man fibrilläres Bindegewebe mit Osteoklasten und leichte Lymphocyteninfiltration. An einer anderen Stelle sicht man ein 2-3 mm breites Knorpelstück, in dessen Umgebung sich neugebildete Knochenbälkchen mit osteoiden Säumen und Osteoblasten befinden.

Das excidicrte Stück der Gclenkkapsel zeigt Stellen mit Lymphocyteninfiltration und weite I.ymphgefäße oder Ausstülpungen der Gelenkkapsel mit riesenzellartigen Bildungen. Keine Anhaltspunkte für 'Tubcrkulose.

Die in Fall 6 excidierten Gewebsteile crgeben folgenden histologischen Befund:

Stückchen Gelenkkapsel mit gut ausgebildeten Zotten. Diese ge- 
fäBreich mit geringerer Lymphocyteninfiltration. Stellenweise kleine Ierde mit Blutpigment, tcils Bindegewebe, teils im Endothel der Zotten. AuBerdem kleine Stückchen. Fettgewebe und Bindegewebe reihen und Osteoidsäume.

Fs lassen sich also, wenn wir unsere Befunde in Fall 2 und 5 kurz zusammenfassen wollen, in denselben unterscheiden: einerseits cine zentrale Nekrose der Spongiosa des Lunatum mit starker Kalkeinlagerung, und andererseits eine Umwandlung der noch crhaltenen Spongiosa im Sinne einer Änderung der Bälkchenstruktur. Aus der auf eine bestimmte Beanspruchung cingestellten radiären Struktur wird ein schwammig angeordnetes Knochengewebe mit größeren oder kleineren Maschen. In Übereinstimmung hiermit finden wir histologisch sowohl Osteoklasten als Zeichen des Knochenabbaues und Osteoblasten mit neugebildeten Osteoidsäumen als Zeichen der Knochenneubildung. Das nekrotische Zentrum ist zum Teil umgeben von einer Zone von Bindegeweben mit Rundzelleninfiltration im Sinne einer demarkierenden Entzündung, zum Teil haftet es der noch crnährten Knochenhülle fest an.

\section{Mechanismus der Verletzung und Natur der Verände- rungen des Lunatum.}

Wenn wir zum richtigen Verständnis der verschiedenartigen Verletzungen des Mondbeines gelangen wollen, so müssen wir zwei Vorkommnisse von vornherein auseinanderhalten:

Erstlich den Bruch bzw. die Zertrümmerung des Lunatum durch eine schwere Gewalteinwirkung, wie sie durch die Fälle von Hunt, Peste, Ricdel, GroB und auch den schon erwähnten Fall der chirurgischen Klinik in Basel erwiesen ist, -eine Verletzung, welche wohl selten isoliert vorkommt, sondern meist als Teilerscheinung anderer schwerer traumatischer Einwirkungen, - und

Zweitens die Formveränderungen des Lunatum, welche dem Arzte erst in chronischen Stadium, Wochen oder Monate nach einer verhältnismäßig leichteren traumatischen Einwirkung zugeführt werden.

Dal. das Lunatum durch ein schweres Trauma gebrochen werden kann, das ist durch die oben angeführten Beobachtungen 
genügend erwiesen. Gewöhnlich erscheint es dabei in zwei bis drei Fragmente zu zerspringen. Dieselben haben keine Neigung, knöchern zusammenzuheilen, sondern es entsteht viclmehr, wie u. a. Pfitzner beschrieben hat, eine Abschleifung der sich berührenden Flächen. Die Fälle sind aber zu selten und bezüglich des genaueren Herganges der Verletzung zu wenig genau bekannt, als daß sich über den Mechanismus irgendwelche bestimmteren Angaben machen ließen. Der Vorgang ist auch bei den besonders in Frage kommenden schweren Verletzungen, wie Sturz aus der Höhe, oft ein recht komplexer und es werden sich wohl manchmal Zug der Bänder und Kompression kombinieren. Diese Fälle haben auch im Hinblick auf ihre Seltenheit und auf die vicl größere Bedeutung der meist vorhandenen übrigen Verletzungen kein geringeres praktisches Interesse, als die folgende Gruppe, zu denen alle 7 Beobachtungen unserer eigenen Arbeit wic überhaupt die meisten in der Literatur mitgeteilten Bcobachtungen von traumatischer Schädigung des Lunatum gehören. An Häufigkeit nehmen dieselben, soweit sich die Frage bis jetzt überblicken läßt, unter den traumatischen Schädigungen des Handwurzelskelettes die vierte Stelle ein. Im ersten Range steht die Fraktur des Naviculare, an zweiter Stelle die sogenannte Luxation des Lunatum, an dritter Stelle die typische intercarpale Luxationsfraktur nach de Quervain-Schoch (- transnaviculoperilunäre Frakturluxation der Hand nach Kienböck) ${ }^{1}$ ).

Es ist nun sehr bezcichnend, daß wir bis jetzt noch kein Röntgenbild dieser leichteren Lunatumveränderungen in frischem Zustande besitzen, so viele Handgelenke auch nach frischen traumatischen Schädigungen mit Röntgenstrahlen untersucht worden sind. Wir können dies nur dadurch erklären, daß die Verletzung anfänglich eine im Röntgenbilde nicht sichtbare Veränderung setzt. Damit ist ohne weiteres gesagt, daß es sich nicht

I) Dic Bezeichnung ,intercarpalpe Luxationsfraktur" stammt nicht, wic K i c n bö ck unrichtig angibt, von v. As s en (1910), sondern von de Q u e r vain, der dic Verletzung schon 1902 als typische Verletzung beschricben und in der I. Auflage seiner chirurgischen Diagnostik Igo7 als inter. carpale L uxationsfraktur bezeichnet hat. Die unter seiner Leitung verfaßte ausführliche Arbeit von $\mathrm{Schoch}$ (1908), welche das ganze, diesbezügliche Material zusammenstellt, ist von $\mathrm{Ki}$ en bö ck übersehen worden. 
um jene schweren traumatischen Verletzungen des Knochens handeln kann, die wir oben besprochen haben, d. h. nicht um wirkliche Frakturen, sonst müßte unbedingt bei der jetzt schon (in halbes Hundert betragenden Zahl von Spätfällen auch einmal cine Lunatumfraktur nach einem geringfügigen Trauma in frischem Zustande zur Beolbachtung gekommen sein. Wir stimmen also Kienböck darin völlig zul, dal3 das Wesentliche an der Verletzung die Ernährungsstörung des Lunatum ist. de Quervain war auf Grund der histologischen Untersuchung bei Fall 2 schon von 1904 zu einer analogen Auffassung gekommen und äuberte sich in scinem Gutachten damals folgendermaßen: „Da die Annahme cines tuberkulösen Hercles sich nicht aufrechtcrhalten läßt, müssen wir annehmen, daß das Zusammengedrücktwerden des Lunatum zur Nekrose der von ihrer Ernährung abgeschnittenen zentralen P'artic gefüht hat."

Diese erste Tatsache der zentralen Ernährungsstörung ergibt sich schon ohne weiteres aus der Betrachtung gewisser Röntgenbilder, wie z. B. von Fall 2 unserer Arbeit und Fall 5, 7, I 2 der Arbeit von Kienböck, um nur die auffallendsten Beobachtungen 7,1 erwähnen. Es grenzt sich in diesen Fällen ein mehr dem proximalen Teil des Lunatum angehöriger Kern als dunkles, für Röntgenstrahlen wenig durchlässiges Crebilde von dem übrigen Lunatum ab, und unsere histologischen Untersuchungen zeigen, daß, wie schon a priori zu erwarten war, dieser Teil nekrotischen Knochen darstellt. Um die nekrotische Partie horum finclet man, wie schon oben crwähnt, stellenweise als Beweis cincr aseptischen demarkierenden Entzündung, eine Zone mit ausgesprochener Rundzelleninfiltration, an welche sich die noch ernährte, periphere Knochenschale anschließ3t, mit Zeichen von Knochenabund -anbau. Die Entstehung dieser Ernährungsstörung läßt sich auf zwei Wegen crklären:

a) Es kann sich um eine Kompressionsfraktur des Lunatum handeln, bei welcher das Mondbein zwischen dem Kopf des Capitatum und der Gelenkfläche des Radius oder bei ciner starken Dorsalflexion gegen eine feste Unterlage so stark gequetscht wird, daß das Balkengefüge des Knochens auch ohne Bruch des Ganzen und ohne auffallende Veränderungen im Röntgenbilde eine Schädigung erleidet. Hierdurch würde die 13lut- 
zufuhr zu den zentralen Teilen desselben unterbrochen, und es würden dieselben infolgedessen der Nekrose anheimfallen, also einer Art Sequestrierung, selbst ohne völlig mechanische Zusammenhangstrennung mit der Umgebung. Die Veränderungen fänden ein Gegenstück in der sogenannten Spondylitis traumatica (von Kümmell). Dies war die Auffassung, welche de Quervain von Anfang an auf Grund der Beobachtung unseres zweiten Falles von der Erkrankung hatte und welcher er auch in seiner "Speziellen chirurgischen Diagnostik“ Ausdruck gab. Ein weiteres Analogon wäre (nach Preiser) ein ähnlicher Vorgang am Naviculare, der auch in einem Falle der chirurgischen Poliklinik in Basel in typischer Weise zur Beobachtung gekommen ist. Auf Grund clieser Ernährungsstörung würde dann der Knochen unter dem Einflusse des Binnendruckes des Handgelenks und der leichteren traumatischen Einwirkungen des täglichen Lebens immer mehr deformiert und würde damit auch nachträglichen traumatischen Schädigungen ernsterer Natur gegenüber weniger widerstandsfähig sein, als ein normaler Knochen. Die im histologischen Bilde beobachteten Abbau- und auch Neubildungsvorgange würden sich durch eine derartige Annahme sehr leicht erklären lassen.

Diese Annahme einer primären Kompressionsfraktur mit anfänglich wenigen auffälligen, aber doch die Ernährung des Knochenzentrums schwer schädigenden Veränderung in der Struktur desselben wurde bis I 910 von allen Autoren vertreten, welche sich mit dieser Frage befaßt hatten. Der sichere Beweis für dieselbe ließe sich allerdings nur durch Fälle erbringen, bei welchen an einem vorher gesunden Handgelenk nach einem nicht allzu schweren Trauma eine wenn auch nur leichte Veränderung der Struktur des Lunatum nachgewiesen werden könnte. Es sind nun allerdings Beobachtungen von Hirsch, Blau, Eberma y er vorhanden, welche diescm Postulat zu entsprechen scheinen. Kienböck macht aber auf Grund eigener Erfahrung den Einwand, daß die betreffenden Fälle keine wirklich frischen waren, d. h. daß schon vor den angeschuldigten Verletzungen Störungen von seiten des Handgelenks vorhanden gewesen seien. Es müßte also in Zukunft genauer als bisher auf diesen Punkt geachtet werden, wenn eine Lunatumveränderung wirklich als 
primär gedeutet werden soll. Dies leitet uns über zu der von Kienböck gegebenen Auffassung:

b) Dic Ernährungsstörung kann bedingt sein durch einen Bändcrabriß am Lunatum. Da die wichtigsten Bänder sich an der Dorsalscite desselben ansetzen, und da sich daselbst auch die meisten Foramina nutritia finden, so müssen auch von dort die wichtigsten Crefälße in den Knochen eintreten. Es müßten also rlurch das Trauma die dorsalen Bänder $s$ weit angespannt werden, dil.3 sic durchreiben bzw. vom Knochen ab. reiben. I iesc Auffassung findet ihre Stütze in folgender Überlegung:

Bei maximaler Dorsalflexion wird das Lunatum, wie die beistehende, aus der Arbeit von Schoch übernommene Fig. I 7 zeigt, völlig nach der Volarseite hin abgetrennt, so daß es die (ielenkfläche des Radius zum größten 'Teil verläßı. Dadurch werden die dorsalen Bandverbindungen in höchstem Grade angespannt. Wird diese Bewegung übortrieben, so muB es zum Abrib der Jorsalbänder kommen und clamit zur Vorstufe der sogenannten ,volaren Luxation des Lunatum". Dieser Bänderabriß würde also einen ersten Akt der Luxation darstellen,

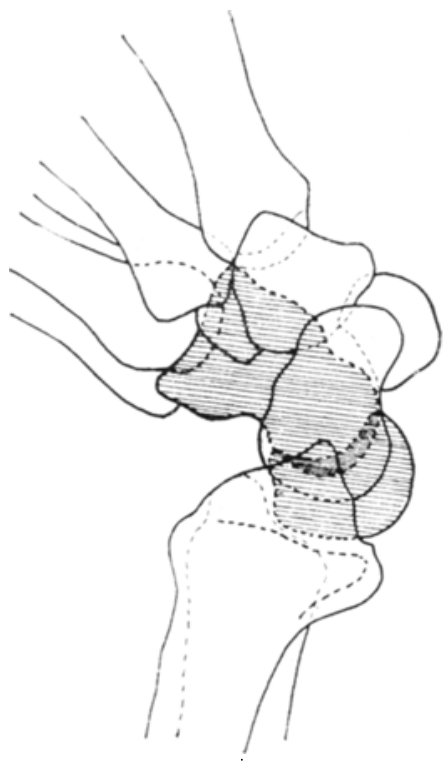

Fig. 17. Dorsale Hyperflexion. Volare Verschicbung des Lunatum.

(Aus Sichoch, Jeutsehe Zcilschr. f. Chir. 1908$, Bd. 91.$)$ bei dem es aber nicht zum Herausschlüpfen des Lunatum aus dem Gelenkverbande gekommen ist. Diese Ansicht Kienböcks wird nun unterstützt durch die weitere Tatsache, daß das Trauma, soweit Angaben über dasselbe vorliegen, meist in einer übertriebenen Dorsalflexion bestanden hat oder wenigstens mit einer solchen verbunden war.

Überschen wir die bisher in der Literatur mitgeteilten und unsere eigenen Beobachtungen, so geht aus denselben zweifellos hervor, daß in einer großen Zahl von Fällen die erstgegebene 
Erklärung der Kompressionsfraktur nicht wohl in Betracht kommen kann, indem eine wirkliche Kompression des Knochens nicht stattgefunden hat. Es gilt dies in unserer eigenen Statistik von den Fällen I und 5. Auch bei den übrigen Fällen ließen sich dic Erscheinungen durch den dorsalen Bänderriß erklären, wenn schon bei denselben die Kompressionsfraktur nicht so bestimmt ausgeschlossen werden kann.

Die weiteren Folgeerscheinungen der Ernährungsstörung -.welches auch ihr Mechanismus sei - - bestehen entweder bloß in allmählichem Schwund des Knochens, also im wesentlichen in resorptiven Vorgängen, wie sie den Bildern unseres Falles I und aus einzelnen Abbildungen von Kienböck hervorzugehen scheinen, zum Teil aber in einer Nekrose mit Osteosklerose der zentralen Partie des Knochens, besonders in scinem proximodorsalen Anteile, und in Umbildungsvorgängen an den ihrer Ernährung noch nicht völlig beraubten Partien, besonders an der Volarpartie. Dort ist ja auch die Bandverbindung nicht unterbrochen, so claß der volare Anteil des Lunatum stets noch ernährt bleibt. Nur ist seine Funktion durch den dorsalen Bänderabriß und die daherige Umgestaltung des dorsalen Knochenabschnittes cine völlig veränderte geworden, und demnach erleidet auch die Knochenstruktur des noch ernährten Teiles einen völligen Umbau. Dabei mögen wiederholte kleinere in physiologischen Grenzen liegende Traumen mitbestimmend wirken, und auch vielleicht zu der Fragmentierung des noch crnährten Knochens ein weiteres beitragen. Eine derartige Auffassung wird uns ganz besonders durch das beim 4. Falle von dem Präparate aufgenommene Röntgenbild nahegelegt. Hier zcigt ein Teil des proximo-dorsalen Stückes noch Ernährung und Balkenstruktur, der größte Teil dagegen Nekrose mit Osteosklerose. Der volare, bei der Operation noch in guter Bandverbindung befindliche und demnach ernährte Anteil zeigt keinerlei nekrotische Veränderungen, wohl aber einen völligen Umbau seiner Bälkchenstruktur und eine Fragmentierung in zwci größere und mehrere kleinere Fragmente. Daß alle diese Vorgänge nicht unmittelbar auf die bloße Anstrengung des Handgelenks bei der Eröffnung einer Konservenbüchse zurückzuführen sind, das dürfte wohl außer Zweifel stehen. 
Lis ließe sich nun weiterhin fragen, ob derartige Vorgänge ihre Analoga an anderen Stellen des Körpers finden. Ls müßte sich um verhältnismälbig isolierte Knochen handeln, welche ihre Ernährung nur von umschricbener Stelle her erhalten und bei denen dicselbe durch einen traumatischen Vorgang zum Teil ausgeschaltet werden kann. Als solcher Knochen ist im Handgelenk vor allem das Naviculare zu erwähnen, wenn schon seine Ernährungsverhältnisse etwas bessere sind als dicjenigen des Lunatum. Wenn man berïcksichtigt, daß, wenn auch nur selten, isolierte Luxationen des Naviculare beobachtet worden sind, so ist es auch sehr wohl denkbar, daß vielleicht noch öfters das Naviculare wenigstens teilweise aus seinen Bandverbindungen herausgerissen wird und, auch ohne luxiert zu werden, schwere Ernährungsstörungen erleidet, und wir sind deshalb mit $\mathrm{Kicn}$ böck der Ansicht, dab die von Preiser am Naviculare beschriebenen Beobachtungen sich zum Teil auch im Sinne der Kie:1böckschen Auffassung crklären lassen.

Ein weiterer Skeletteil, der unter ähnlichen Bedingungen steht, ist der Schenkelkopf. Er erhält allerdings seine Emährung vom Halse her, zum Teil aber doch durch das Ligamentum teres. Nach Prof. de Q uervain liegt es sehr nahe, gewisse, allmählich eintretende Ernährungsstörungen des luxiert gewesenen Schenkelkopfes auf die Zerreißung des Ligamentum teres zurückzuführen. I amit ließ3en sich dieselben olne weiteres mit den uns hier beschäftigenden Lunatumveränderungen in Parallele stellen. Dasselbe gilt nach seiner Auffassung von der von Perthes beschriebenen deformierenden Ostitis des (Oberschenkelkopfes im Wachstumsalter.

\section{Prognose.}

Auch bei der Besprechung der I'rognose müsses wir die beiden vorhergehenden beschriebenen Formen auseinanderhalten, nämlich die cigentliche Fraktur mit Bildung ron zwei bis drei Fragmenten und die auf Kompression bzw. Bänderabriß zurückzuführende Jirnährungsstörung mit sekundärer Fragmenticrung.

Was die eigentliche Fraktur des I,unatum betrifft, so müssen wir aus den bisher vorliegenden $A$ ngaben schlie 3 en, dalis dieselbe keine Neigung zu knöcherner Heilung zeigt. Die Bruchstücke 
bleiben viclmehr, wie wir das vom Naviculare ja auch kennen, jahrelang unvereinigt, ja sic schleifen sich gegenseitig ab. Der Hauptgrund des Ausfallens der Callusbildung ist wohl das Fehlen von Periost. Der größte Teil der Oberfläche des Lunatum besteht aus Knorpel, und die beiclen Bänderansätze bilden keinen genügenden Ausgangspunkt für Callusbildung. Wenn gar ein drittes, keinen Bänderansatz tragendes Fragment entsteht, so muß dasselbe notwendig die Rolle eines allerdings wenig beweglichen Gelenkkörpers spiclen. Die weitere Folge dieses Vorganges ist die Entstchung eines gewissen Grades von chronischer deformierender Arthritis. Daß solche Fälle jahrelang Beschwerden aufweisen können und daß aus der Verletzung eine selbst. bleibende Verminderung der Gebrauchsfähigkeit der Hand entsteht, das liegt auf der Hand. Ein gutes Beispiel hierfür liefert der Fall Groß.

Viel schwieriger zu beurteilen ist die Prognose bei den. Fällen der zweiten Gruppe, denjenigen der Ernährungsstörung des Lunatum ohne primäre Fragmentierung desselben. Da dem Arzte nur diejenigen Fälle zugehen, welche Beschwerden aufweisen, so wissen wir nicht, wie oft die Störungen sich wieder verlieren. Wic bei so vielen Erkrankungen macht das Kontingent der nichtdiagnostizierten Fälle die Prognose in Wirklichkeit zu einer besseren, als sie uns erscheint, wenn wir nur die sicher erkannten Fälle unserem Urteil zugrunde legen. Diese Lücke in unserem Wissen könnte einigermaßen ausgeglichen werden durch jahrelang fortgesetzte Beobachtung der diagnostizierten, aber nicht operativ behandelten Fälle. Eine solche Beobachtung liegt aber bis jetzt für die wenigsten in der Literatur mitgeteilten. Lunatumschädigungen vor.

Auch in den Fällen, bei welchen die Anamnese auf Jahre zurückgreift, fehlen bis jetzt in größeren Intervallen aufgenommene Röntgenbilder. Hier tritt Fall I unserer Arbeit in die Lücke, indem die Röntgenbilder im Intervall von $\mathrm{I}^{1} / 2$ Jahren aufgenommen wurden. Nachdem auf die einleitende Behandlung (Jodoform-Karbolinjektionen) eine Besserung eingetreten war und nachdem eine Nachuntersuchung nach 5 Jahren subjektiven Wohlbefindens ergeben hatte, zeigt doch die nach $\mathrm{I} I 1 / 2$ Jahren vorgenommene Nachuntersuchung, daß die Beschwerden, wenn auch 
weniger ausgesprochen als im Beginn, doch noch fortbestehen und der Patientin bei gewissen Handarbeiten beschwerlich fallen. Die Röntgenuntersuchung ergibt sogar, wic oben auscinandergresetzt, das Vorhandensein von deutlichen deformicrenden Veränderungen an der Gelenksfläche des Radius und einer Andeutung von solchen an allen Knochen des Radiocarpalgelenks uberhaupt, während das Intercarpalgelenk und das Carpo-Metacarpalgelenk völlig frei ist, vom Capitatum abgesehen. 1)ab es sich dabei nicht ctwa um eine Teilerscheinung einer allgemcinen deformierenden Arthritis landelt, das beweist die Tatsache, daß die übrigen Gelenke und ganz besonders das andere Handgelenk völlig normal ist. Es ergibt sich hieraus, daß dic l'rognose der verlassenen Lunatumveränderung, auch wenn sie nie zu schweren Störungen fülıren sollte, doch nicht allzu günstig zu stcllen ist und daß nicht nur kein Rückgang der Veränderungen des Lunatum, sondern cher noch eine gewisse Mitbeteiligung der Nachbarknochen in Aussicht genommen werden muB. Hiergegen kann nichts angeführt werden, dal3 in Fall $7 \mathrm{I} / 2$ Jahre nach der Verletzung dic Arbejtsfähigkeit nicht cingeschränkt ist. Iis ist nicht ausgeschlossen, daß in diesem lalle späterhin noch Störungen eintreten werden. In Fall 6 war die $A$ rbeitsfïhigkeit nach 5 Jahren noch normal, doch war der fall auch anatomisch ein leichter. Leicler war keine Nachuntersuchung möglich. Auch jn Fall 16 von Kienböck bestanden moch nach r. Jahren crnstliche Beschwerden, dann schwanden diescllyen aber wieder völlig, sehr wahrscheintich, um später wieder in die Erscheinung zu treten. So wünschenswert es auch wäre, schon jet»t zu Händen der Beurteilung der Arbeitsfähigkeit bei versicherten Arbeitern grenaue Angaben machen zu können, so unmöglich ist cs für den Augenblick, erst die weitere Beobachtung einer größßeren Anzahl von Fällen wird dies uns möglich machen, und licerzu wird die Gelegenheit in Zukunft öfter greboten sein, sobald cinmal die Erkenntnis dieser Erkrankung Gemeingut der Ärzte geworden ist. Vorläufig müssen wir uns damit beschciden, in den nichtoperierten Fällen die Wahrscheinlichkeit oder allermindestens die Möglichkeit jahrelang dauernder leichter I'unktionsstörung vorauszuschen. Auch dann, wenn wir Grund zu haben glauben, eine bleibende Verminderung der Arbeitsfähigkeit an- 
zunehmen, läßt sich dics aber nicht nach irgendeinem Schema berechnen, sondern wir müssen in jedem einzelnen Falle die Intensität der Schmerzen, den Ausfall der Bewegungsausschläge, die besondere Arbeit des Verletzten und nicht zum mindesten sein Niter in Rücksicht ziehen.

Die Prognose der operierten Fälle werden wir weiter unten besprechen.

\section{Behandlung.}

Auch für die Behandlung sind die beiden Hauptformen auscinanderzuhalten.

Ist das Lunatum primär in zwei oder mehrere Stücke gebrochen, so werden wir uns, wenn ernstliche Beschwerden bestchen, ohne Schwierigkeiten entschließen, dasselbe zu cntfernen. Wir dürfen uns dabei jedoch nicht verhehlen, daß durch den Kingriff die Statik und Mechanik des Gelenks nicht in geringem Grade beeinträchtigt wird, und wir sehen denn auch, daß sich nach der Operation die Funktionsfähigkeit selbst bei korrekter Nachbehandlung nicht immer sehr rasch wicderherstellt. Daß das Gelenk aber mit der Zeit doch wieder gebrauchsfähig wird, das können wir aus unseren eigenen Beobachtungen (Fall 2 und 4) crsehen, wenn dieselben schon Verletzungen des zweiten Typus betreffen.

Bei diesem zweiten Typus, der Ernährungsstörung des Lunatum ohne primäre Fraktur in gewöhnlichem Sinne, werden wir uns weniger leicht zur Entfernung des geschädigten Knochens entschließen. Jedenfalls werden wir konservativ vorgehen, wenn das Röntgenbild uns zeigt, daß das Lunatum nicht schwer verändert ist. Eo ipso gilt dies von dem Anfangsstadium der Verletzung, in dem übrigens, wie wir oben schon gesehen haben, dic Diagnose viclleicht selbst im Röntgenbilde noch nicht gestellt werden kann, und in dem also der Fall einfach in die Kategorie der Handgelenksdistorsionen eingereiht und als solche mit Massage und vorübergehender Ruhigstellung des Gelenks, aber keinesfalls mit Operation behandelt wird. Gerade in diesen Fällen leisten Karbolinjektionen (Einspritzung von $\mathrm{I}-2 \mathrm{~cm}^{3}$ cincr I- -2 proz. [nicht zu alten!| Karbol- oder Orthokresollösung 2 mal in der Woche) oft recht gute Dienste. Der Karbolkomponente 
Der Kompressionsbruch und die traumatische Erweichung des Mondbeines. I 6 I

können wir dann auch wohl die ziemlich rasche Besserung der Symptome in Fall I zuschreiben. Auch Heißluftbehandlung oder irgendeine andere Form aktiver Hyperämie wird sich als nützlich erweisen können. Kommen wir so zu keinem Ziele und werden die Strukturveränderungen des Lunatum im Röntgenbilde immer ausgeprochenere, oder sind sie schon bei der ersten Untersuchung sehr ausgesprochene, so werden wir die Entfernung des Lunatum in Betracht ziehen müssen. Auch hier gilt wieder das oben Gesagte, daß mit der Operation die Funktion des Gelenks noch nicht sofort wiederhergestellt ist, sondern daß3 wir noch mit einer längeren Periode der Nachbehandlung rechnen müssen. Wir machten dieselbe Beobachtung schon seit Jahren bei der Exstirpation des I-unatum wegen Luxation bzw. wegen intercarpaler Luxationsfraktur, wie dies in der Arbeit von Schoch des genaueren auseinandergesetzt ist. Sind die Veränderungen des Knochens bei der ersten Untersuchung schon ausgesprochene, so wird es sich wohl empfehlen, den Eingriff nicht allzu weit hinauszuschieben, damit nicht in der Umgebung die İrscheinungen einer chronischen traumatischen Arthritis auftreten, welche ihrerseits die Rekonvaleszenz zu einer außerordentlich langen machen könnten. Es hat sich ja, wie de Q uervain schon gezeigt hat, gerade bei der intercarpalen Luxationsfraktur herausgestellt, daß diejenigen Fälle am raschesten wieder funktionsfähig werden, bei denen der Eingriff am frühesten nach der Verletzung ausgeführt worden ist.

İber die Operation selbst ist nicht viel Besonderes beizufügen. Ein dorsaler Schnitt führt ohne weiteres auf das Lunatum und dic einzige Schwierigkeit kann in der Entfernung des vielleicht abgetrennten oder mit dem proximo-dorsalen Fragment nur sehr locker verbundenen volaren Iragmente bestehen. Frühe Wiederaufnahmen der Bewegungen nach dem Eingriffe wird die Rekonvaleszenz beschleunigen. Ganz besonders sind die Fingerbewegungen von Anfang an, auch im Verbande, zu üben. Daß die Hand in leichter Dorsalflexion eingebunden werden muß, das braucht kaum hervorgehoben zu werden. Zur Beurteilung der Fernresultate kommen unsere Fälle 2 und 5 in Betracht. Vergleichen wir das erzielte Resultat mit dem Zustande des nichtoperierten Falles I, bekommen wir den Eindruck, 
daß der Vorteil eher auf sciten der Operation liegt, wennschon die Entfernung des Lunatum die Ausbildung leichter deformierender Veränderungen nicht hintanhalten konnte.

Eine bestimmtere Indikationsstellung wird sich allerdings, wie die sichere Beurteilung der Prognose, erst aus den Erfahrungen ergeben, die über das hier beschriebene Verletzungsbild noch gewonnen werden müssen.

\section{Schlußsätze.}

Wir können den Inhalt unserer Arbeit kurz in folgende Sätze zusammenfassen:

I. Neben den seltenen, nur durch schwere Verletzung zustande kommenden primären Frakturen des Mondbeines gibt es eine Katcgorie von traumatischen Schädigungen desselben, welche auf traumatisch bedingte Ernährungsstörungenzurückzuführen sind.

2. Entweder handelt es sich dabei um die Folge einer Kompressionsfraktur mit teilweiser Unterbrechung der Blutzirkulation im Innern des Knochens oder um einen zu schweren Zirkulationsstörungen führenden dorsalen Bänderabriß.

3. Die erstere Deutung wird durch gewisse Röntgenbeobachtungen nahegelegt und erscheint für eine Anzahl von Fällen als wahrscheinlich. Eine noch größere Zahl läßt sich aber viel ungezwungener durch die zweite Hypothese (Kienböck) erklären.

Von den 7 in unserer Arbeit beschriebenen Fällen lassen sich 4 durch die eine wie die andere Hypothese erklären, während bei 3 derselben ein Bandabriß anzunchmen ist.

4. Unsere beiden histologisch untersuchten Fälle zeigen eine zentrale Nekrose des Lunatum mit starker Kalkeinlagerung und demarkierender Rundzelleninfiltration und eine periphere noch ernährte Knochenschale mit Umbau der Spongiosa.

5. Die Symptome sind zuerst diejenigen einer gewöhnlichen Distorsion: Schmerz, Schwellung, Funktionsstörung. Erst das Andauern oder die häufige Wiederkehr derselben, mit Sitz des Schmerzes an der Dorsalseite des Lunatum, läßt an die isolierte traumatische Schädigung dieses Knochens denken.

6. Für die Differentialdiagnose kommt neben den übrigen Handgelenksverletzungen besonders die Tuberkulose in Frage. Verwechslungen werden allerdings, seitdem das Verletzungsbild 
der traumatischen Erweichung des Lunatum bekannt geworden ist, immer seltener werden.

7. Die Prognose der primären Frakturen des Lunatum ist quoad consolidationem eine ungünstige. Diejenige der traumatischen Erweichung läßt sich deshalb nur schwer aufstellen, weil wir das frische Verletzungsbild nicht mit Sicherheit diagnostizieren können und deshalb kein Urteil darüber haben, in wieviel Fällen der Abriß der dorsalen Bänder wirklich zu der schweren Formveränderung des Knochens führt, wie dies uns in den bisher beschriebenen Fällen nachträglich zu Gesichte kommt. Ist die Veränderung des Lunatum einmal ausgebildet, so kann sie zu umschriebener deformierender Arthritis des Radiocarpalgelenks und zu jahrelang dauernden, ja bleibenden Funktionsstörungen des Gelenks führen.

8. Bei sicheren primären Frakturen des Lunatum, wo dic Entfernung des geschädigten Knochens erwogen werden muß, darf bei der Unsicherheit der Prognose in Fällen von beginnender traumatischer Erweichung länger zugewartet werden. Öfter wird abcr auch hier die Entfernung des Lunatum angezeigt sein, um die gestörte Handgelenksfunktion wieder zu einer annähernd normalen zu machen. Die Entfernung des Lunatum schließt aber die nachträgliche Entstehung von deformicrenden Veränderungen nicht aus. Eine genaue Indikationsstellung wird sich erst auf Grund wciterer Erfahrungen geben lassen.

\section{Nachtrag.}

Nach Abschluß dieser Arbeit erschien die ausführliche Mitteilung der Beobachtung von Baum${ }^{1}$ ) und ferner die Mitteilung von zwei Fällen mit histologischem Befund aus der Züricher Klinik durch H. C. Frenkel-Tissot ${ }^{2}$ ). Dieselben enthalten interessantes Vergleichsmaterial, ergeben aber keine neuen Anhaltspunkte für die noch schwebenden Fragen.

I) L. W. I3 a u m, über die tarumatische Affektion des Os lunatum und navicularc carpi. Bcitr. z. klin. Chir., Bd. 87, S. 568.

2) H. C. Irenkel-Tissot, Beiträge zur Frage der traumatischen Ernährungsstörung des Os lunatum manus. Fortschritte auf dem Gebiete der Röntgenstrahlen, Bd. 21, S. 536 . 


\section{Literaturverzeichnis.}

I. Bardenheuer, Die Verletzungen der oberen Extremitäten. Teil II. Deutsche Chirurgie I888, S. 238.

2. B a u m, Über die sogenannte Ostitis des Os lunatum. Zentralbl. f. Chir. 1913, S. 1405 .

3. Beck, C., Die Röntgenstrahlen im Dienste der Chirurgie. Teil I. München I902.

4. Blau, O., Fünfzehn Kahnbcinbrüche. Fractura ossis nav. carpi. Deutsche Zeitschr. f. Chir. 1904, Bd. 72, S. 445.

5. Bri ot et Destot, Anatomie et physiologic du poignet par la radioscopie et la radiographie. Prov. méd. I898, p. 97 et Io9.

6. Bryce, Mécanisme du poignet. Journ. of Anat. and Physiol. London I 896-97.

7. Codman et $\mathrm{Chase}$, The Diagnosis and treatment of fracture of the carpal scaphoid and dislocation of the semilunar bone. Annals of surg. 1905, März u. Juni, Nr. 315. Ref. in Zentralbl. f. Chir. 1905.

8. Cous in, De l'emploi de la radiographie dans les lésions traumatiques du poignet. Thèse de Lyon 1897.

9. Destot, Scaphoide et fracture du poignet. Iyon $1898, \mathrm{XXX}, 448$.

ıo. - Recherches radiographiques sur les fractures du poignet. Echo méd. Lyon 1898 , I 5 .

I I. - Lésions traumatiques du poignet. Verhandlungen der Deutschen Röntgengesellschaft i905, III. Teil, S. 224.

12. - Le poignet et les accidents du travail. Etude radiographique et clinique, Paris 1905 .

13. Destot et Gallois, Recherches physiol. et expérim. sur les fractures de l'extrémité inférieure du Radius. Revue de Chir. I898, p. 886.

14. D u m s r ey, Verletzungen der Handwurzel, durch Diagraphie diagnostiziert. Monatsschr. f. Unfallheilk. $1898, \mathrm{H}, 3$.

I5. E b e r m a er, F., Über isolierte Verletzungen der Handwurzelknochen. Fortschritte auf dem Gebiete der Röntgenstrahlen 1908, Bd. I2, S. I.

I6. Eigenbrodt, Über isolierte Luxationen der Carpalknochen. Bruns' Beitr. z. klin. Chir. 190I, Bd. 30, S. 805 .

I7. Friedrich, P. L., Verletzungen und Erkrankungen des Handgelenks. Handb. d. prakt. Chir,, 2. Aufl.

I8. Gallo is, Fractures de l'extrémitć inférieure du Radius. Thèse de Lyon 1898.

19. Gocht, Seltenere Frakturen in Röntgenscher Durchleuchtung. Mitteil. aus den Hamburger Staatskrankenanstalten I897, II, 24 I.

20. Grashey, R., Atlas chirurg.pathologischer Röntgenbilder. Lehmanns med. Atlanten, N. F., I908, Bd. 6.

2I. - Fortschritte auf dem Gebiet der Röntgenstrahlen, Bd. II, H. 3.

22. GroB, H., Der Mechanismus der Luxatio ossis lunati. Arch. f. klin. Chir. 1903, Bd. 7o, S. 793. 
Der Kompressionsbruch und die traumatische Erweichung des Mondbeines. 165

23. Gruber, Os naviculare bipartitum. Virchows Arch. 1877, Bd. 69, S. 391 .

24. Hirsch, M., Über isolicrt subkutane Frakturen einzelner Handwurzelknochen. Deutsche Zeitschr. f. Chir. 1907, Bd. 86, S. 135 und briefliche Mitteilung über dic darin ziticrten Fälle.

25. -- Über Fraktur des Os naviculare. Fortschritte auf dem Gebiete der Röntgenstrahlen I9I0, Bd. I 5, S. 22 r.

26. Hö flinger, J., Uber Frakturen und Luxationen des Carpalknochens. Korrespondenzbl. f. Schweizer Ärzte Igor, Bd. 31, S. 297.

27. H $\mathrm{unt}$. Annals of anat. and surg. I $88 \mathrm{I}$, t. III, p. Iro.

28. Kahleys, Beitrag zur Kenntnis der Frakturen am unteren Ende des Radius. Deutsche Zeitschr. f. Chir. 1897.

29. Ka ufmann, Zur Verwendung der Röntgenschen Skiagraphic bei der Begutachtung von Verletzten. Monatsschr. f. Unfallheilk. I896, S. 258.

30. Ki enb $\ddot{c} \mathrm{ck}$, R., Über traumatische Malacie des Mondbcins und ihre Folgezustände. Fortschritte auf dem Gebicte der Röntgenstrahlen, Bd. I6, S. 77.

3r. Körte, Fall von Luxation der Hand nach hinten mit Aussprengung des Os Iunatum. Deutsche med. Wochenschr. 1898, Ver.-Beil., S. 3 I.

32. L a bady, Fitude sur les luxations médiocarp. traumatiques. Thèse de Paris 1897 , p. 58.

33. L a u enstein, Luxation des Os lunatum nach der Vola zu nebst Fraktur des Proccssus styloideus von Radius und Ulna. Fortschritte auf dem Gebiete der Röntgenstrahlen I898, Bd. 2, S. 2 I 8.

34. Lilienfeld, A., Dic Luxatio ossis lunati volaris, eine Luxatio ossis capitati dorsalis. Arch. f. klin. Chir. 1905, Bd. 76, S. 64I.

35. - Uber die Carpalia usw. Fortschritte auf dem Gebicte der Röntgenstrahlen I908-09, Bd. I3, S. 133 .

36. Mougeot, Malgaigne, Traité des fractures et des luxations 1855, II, p. $68 \mathrm{r}$.

37. Morin, Radiographie et entorse du poignet. Gaz. méd. de Nantes I $898, \mathrm{IV}, 27$.

38. Natvig, Tidskrift for den horske Lägeforeming 1901, p. 339. Ref. Zentralbl. f. Chir.

39. - Brüche der Ifandwurzelknochen. Monatsschr. f. orthop. Chir. u. physik. Heilunethoden 1903, S. 125.

40. Nion, Uber Handwurzelknochenbrüche. Deutsche milit. Zeitschr. I903, 198. Ref. in Monatsschr. f. Unfallheilk. I903, S. 290.

4I. Oberst, Brüche der Handwurzelknochen. Arch. u. Atlas d. norm. u. patholog. Anatomic in Röntgenbildern, Ergänzungsheft 5, S. 9.

42. - Frakturen und Luxationen. I. Teil. Erg:inzungsband $V$ zu lortschritte aut dem Gebiete der Röntgenstrahlen igor, S. Io.

43. Pfitzner, W., Beiträge zur Kenntnis des Extremitätenskeletts. Morpholog. Arbeiten (Schwalb c) I 895, Bd. 4, S. 347.

44. - Dic morphologischen Elemente des menschlichen Hanclskeletts. Zeitschrift f. Morphologie u. Anthropologie 1900, Bd. 2, S. 77 u. 565. 
I 66 GUYE, Der Kompressionsbruch u. d. traumat. Erweichung d. Mondbeines.

45. P este, M. Bulletin de la Société anatomique de Paris 1843 , vol. XVIII. 46. Preiser, G., Über posttraumatische Ostitis des Naviculare. I'ortschritte auf dem Gebiete der Röntgenstrahlen I9Io, Bd. 4, S. I89.

47. Quervain, de F., Beitrag zur Kenntnis der kombinierten Frakturen und Luxationen der Handwurzelknochen. Monatsschr. f. Unfallheilk. 1902 , Bd. 9, S. 65.

48. - Spezielle chirurgische Diagnostik. IV. Leipzig 19r3, A. Vogel.

49. Reinhardt-Natvig, Brüche der Handwurzelknochen. Monatsschr. f. orthop. Chir. u. physik. Heilmethod. I902, Nr. Io. Ref. in Monatsschr. f. Unfallheilk. 1903, S. 390.

5o. Schmiz, Über Frakturen und Luxationen der Handwurzelknochen. Deutsche militärärztl. Zeitschr. 1902, Bd. 3I, S. 3rr.

5I. Schoch, V., Beitrag zur Kenntnis der typischen Luxationsfraktur des Intercarpalgelenks.

52. S te in man n, Handwurzelverletzung. Korrespondenzbl. f. Schweizer Ärzte 1904, Bd. 34, S. 628 .

53. T a a f e c, Dislocation of the semilunar bone of the carpus. Brit. med. Journal 1869 , I, 398.

54. Thilenius, Die überzähligen Carpalien menschlicher Embryonen; vorläufige Mitteilung. Anat. Anzeiger 1894, S. $665 \mathrm{ff}$.

55. Th o ele, Zur Kasuistik seltener Luxationen und Frakturen. Deutsche Zeitschr. f. Chir. Ig00, 8, IIz.

56. T u rner, W., Variations of the Carpus. Journal of anatomy and physiology i 883 , XVII, p. 244.

57. Vollrath, Über Handverstauchungen. Verein der Sanitätsoffiziere der Garnison Stettin, 9. XII. 1904. Ref. Fortschritte auf dem Gebiete der Röntgenstrahlen, Bd. 9, S. 67.

58. Witteck, A., Uber Verletzungen der Handwurzel (Os lunatum). Bruns' Beitr. z. klin. Chir. 1904, Bd. 42, S. 578 .

59. W olff, R., Über Handwurzelverletzungen usw. Deutsche Zeitschr. f. Chir. I903, Bd. 70, S. 254 u. 289.

6o. - Über das Röntgenbild der Handwurzel in Hinsicht auf die Luxationsstellung des Handgelenks. Monatsschr. f. Unfallheilk. u. Invalidenwesen I903, X. Jahrg., Nr. 7, S. 2or.

61. - Vortrag in der Freien Vereinigung der Chirurgen Berlins. Deutsche med. Wochenschr. I903, Nr. 43, Vercinsbeil., S. 338.

62. - Frakturen des Os naviculare usw. Deutsche Zeitschr. f. Chir. I903, Bd. 69, S. $40 \mathrm{I}$.

63. - Frakturen des Os naviculare usw. Arch. f. klin. Chir. I905, Bd. 77, S. 634 . 